\title{
LICHTENBAUM-TATE DUALITY FOR VARIETIES OVER P-ADIC FIELDS
}

\author{
JOOST VAN HAMEL
}

ABSTRACT. S. Lichtenbaum has proved in [L1] that there is a nondegenerate pairing

$$
\operatorname{Pic}(C) \times \operatorname{Br}(C) \rightarrow \operatorname{Br}(K)=\mathbf{Q} / \mathbf{Z}
$$

between the Picard group and the Brauer group of a nonsingular projective curve $C$ over a $p$-adic field $K$ (a finite extension of the $p$-adic numbers $\mathbf{Q}_{p}$ ). On the level of divisors the pairing is induced by the norm map $\operatorname{Br}\left(K^{\prime}\right) \rightarrow \operatorname{Br}(K)$ for finite extensions $K^{\prime} / K$. The nondegeneracy is proven by a reduction to Tate duality for commutative group schemes over $p$-adic fields. This reduction is achieved by explicit cocycle calculations in Galois cohomology. The present paper introduces a new homology theory, pseudo-motivic homology, which allows us to reconstruct the above duality as a purely formal combination of a generalised form of Tate duality over $p$-adic fields and a form of Poincaré duality for curves over arbitrary fields due to P. Deligne. This gives a more conceptual proof of Lichtenbaum's result and an analogue in higher dimensions.

\section{Introduction}

Let $\varphi: X \rightarrow \operatorname{Spec} K$ be a variety over a $p$-adic field, and consider the Yoneda pairings

$$
\operatorname{Ext}^{2-i}\left(R \varphi_{*} \mathbf{G}_{m}, \mathbf{G}_{m}\right) \times H^{i}\left(X, \mathbf{G}_{m}\right) \rightarrow \operatorname{Br}(K)=\mathbf{Q} / \mathbf{Z} .
$$

We will see that these pairings gives a good generalisation of Lichtenbaum's pairing between the Picard group and the Brauer group of a curve. Here the Ext-groups should be computed on the smooth site; see Section 1.2 for a definition and a motivation of this choice of topology. These groups give interesting homology groups for varieties over an arbitrary field $k$. For technical reasons we will require that the ground field $k$ has characteristic zero and that $\varphi: X \rightarrow$ Spec $k$ is proper and smooth (see Section 2.3). The analogy to étale homology with coefficients in $\mathbf{Z} / n$ prompts for the notation

$$
{ }^{1} H_{i}(X, \mathbf{Z}):=\operatorname{Ext}_{k_{\mathrm{sm}}}^{-i}\left(R \varphi_{*} \mathbf{G}_{m}, \mathbf{G}_{m}\right)
$$

with the ' 1 ' added in the notation in order to avoid confusion with motivic homology. Indeed, these groups can be regarded as intermediates between étale homology with coefficients in $\hat{\mathbf{Z}}$ and motivic homology with coefficients in $\mathbf{Z}$. For example, when $k$ is algebraically closed, we have for $i>2$ that

$$
{ }^{1} H_{i}(X, \mathbf{Z})=H_{i}(X, \hat{\mathbf{Z}}),
$$

whereas ${ }^{1} H_{0}(X, \mathbf{Z})$ is canonically isomorphic to (the $k$-points of) the total Albanese variety of $X$ (see Sections 1.1, 2.2, and 3.2). On the other hand, the motivic homology group

1991 Mathematics Subject Classification. 14G20 (Algebraic geometry — Local ground fields), 14F22 (Brauer groups of schemes), 11S25 (Algebraic number theory: local and $p$-adic fields — Galois cohomology), 14C25 (Algebraic cycles). 
$H_{0}(X, \mathbf{Z})$ is the Chow group of zero-cycles. Therefore I will refer to the homology theory defined above as pseudo-motivic homology. The following result shows that for duality over a $p$-adic field these pseudo-motivic homology groups are just right.

Theorem 1. Let $X$ be a smooth proper variety over a finite extension $K$ of $\mathbf{Q}_{p}$. For every $r \in \mathbf{Z}$ the Yoneda pairing

$$
{ }^{1} H_{r}(X, \mathbf{Z}) \times H^{r+2}\left(X, \mathbf{G}_{m}\right) \rightarrow \operatorname{Br}(K)=\mathbf{Q} / \mathbf{Z}
$$

is nondegenerate, inducing perfect pairings

$$
\begin{array}{rrr}
{ }^{1} H_{r}(X, \mathbf{Z}) \times H^{r+2}\left(X, \mathbf{G}_{m}\right)^{\wedge} \rightarrow \mathbf{Q} / \mathbf{Z} & \text { for } r=-2,-1, \\
{ }^{1} H_{r}(X, \mathbf{Z})^{\wedge} \times H^{r+2}\left(X, \mathbf{G}_{m}\right) & \rightarrow \mathbf{Q} / \mathbf{Z} & \text { for } r=0,1,
\end{array}
$$

and

$$
{ }^{1} H_{r}(X, \mathbf{Z}) \times H^{r+2}\left(X, \mathbf{G}_{m}\right) \rightarrow \mathbf{Q} / \mathbf{Z} \quad \text { for } r \geq 2 .
$$

Here a pairing between topological groups $A \times B \rightarrow \mathbf{Q} / \mathbf{Z}$ is called nondegenerate if the induced homomorphisms from $A$ to the Pontryagin dual of $B$ and from $B$ to the Pontryagin dual of $A$ are monomorphisms and perfect if these induced maps are isomorphisms. The topology we choose implicitly on our groups is the discrete topology for torsion groups and the profinite topology on all other groups. The notation $A^{\wedge}$ denotes the completion of $A$ with respect to the profinite topology.

Proof. For $X$ geometrically irreducible, this is a special case of Theorem 4.3; removing the irreducibility condition is a straightforward generalisation.

The following result, due to P. Deligne, relates Theorem 1 to Lichtenbaum-Tate duality for curves.

Theorem (Poincaré duality for curves). Let $C$ be a smooth projective curve over a field of characteristic zero. For any $i \in \mathbf{Z}$ we have a natural isomorphism

$$
H^{i}\left(C, \mathbf{G}_{m}\right) \stackrel{\sim}{\rightarrow}{ }^{1} H_{1-i}(C, \mathbf{Z}) .
$$

Proof. This follows from [SGA4, XVIII.2]. See Section 3.3.

Hence we obtain a new, more conceptual proof of Lichtenbaum's result.

Corollary (Lichtenbaum-Tate duality [L1]). Let $C$ be a smooth projective curve over a $p$-adic field $K$. For every $i \in \mathbf{Z}$ we have a nondegenerate pairing

$$
H^{i}\left(C, \mathbf{G}_{m}\right) \times H^{3-i}\left(C, \mathbf{G}_{m}\right) \rightarrow \mathbf{Q} / \mathbf{Z} .
$$

These pairings satisfy the usual symmetry rules for cup products, and they induce perfect pairings

$$
\begin{aligned}
& H^{0}\left(C, \mathbf{G}_{m}\right)^{\wedge} \times H^{3}\left(C, \mathbf{G}_{m}\right) \rightarrow \mathbf{Q} / \mathbf{Z}, \\
& H^{1}\left(C, \mathbf{G}_{m}\right)^{\curlywedge} \times H^{2}\left(C, \mathbf{G}_{m}\right) \rightarrow \mathbf{Q} / \mathbf{Z} .
\end{aligned}
$$

PROOF. The existence, nondegeneracy and perfectness of the pairings follows immediately from the theorems above. The symmetry follows from the construction (see Theorem 3.7). The implicit claim that the pairings given here coincide with Lichtenbaum's pairings is justified by Lemma 3.1. 
Note that the proof of the duality for curves by combining Tate duality in Galois cohomology and Poincare duality in sheaf cohomology is analogous to the canonical way of obtaining a perfect duality

$$
H^{i}\left(X, \mu_{n}^{\otimes j}\right) \times H^{2 d+2-i}\left(X, \mu_{n}^{\otimes d+1-j}\right) \rightarrow \mathbf{Z} / n
$$

in the étale cohomology with torsion coefficients of a nonsingular proper variety $X$ of dimension $d$ over a $p$-adic field (cf. [Sa], compare [M2, Th. II.1.16]).

\section{Application to Roquette's problem}

One of Lichtenbaum's original motivations for establishing his duality theorem was to get a better understanding of Roquette's result that for an irreducible curve $C$ over a $p$-adic field $K$ the order of the kernel of the natural map

$$
\operatorname{Br}(K) \rightarrow \operatorname{Br}(K(C))
$$

is equal to the index $I(X)$ of $C$, which is by definition the index of the image of the degree $\operatorname{map} \operatorname{Pic}(X) \rightarrow \mathbf{Z}$ ([Ro, Th. 1], see also [L1, p. 120]). Theorem 1 allows us to give a dual description to the kernel of the analogue of (2) for higher dimensional varieties.

Theorem 2. Let $\varphi: X \rightarrow \operatorname{Spec} K$ be a smooth projective irreducible variety over a $p$-adic field. The kernel of the canonical map

$$
\operatorname{Br}(K) \rightarrow \operatorname{Br}(K(X))
$$

is a finite cyclic group dual to the cokernel of the degree map

$$
\varphi_{*}:{ }^{1} H_{0}(X, \mathbf{Z}) \rightarrow{ }^{1} H_{0}(\operatorname{Spec} K, \mathbf{Z})=\mathbf{Z},
$$

Proof. The map (3) factorises via the cohomological Brauer group $\operatorname{Br}(X):=H^{2}\left(X, \mathbf{G}_{m}\right)$, which injects into $\operatorname{Br}(K(X))$. The projection formula (12) gives us a compatible diagram of nondegenerate pairings

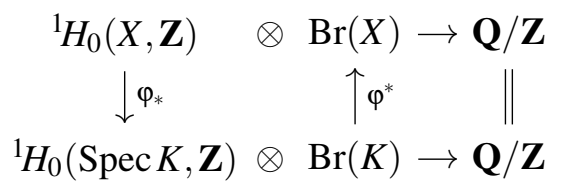

Both pairings are nondegenerate by Theorem 1 .

Of course this result is not quite as attractive as Roquette's result for curves. It would have been nicer to get the index $I(X)$ of $X$ (the index of the image of the degree map $\left.\mathrm{CH}_{0}(X) \rightarrow \mathbf{Z}\right)$, rather than the index of the map ${ }^{1} H(X, \mathbf{Z}) \rightarrow \mathbf{Z}$, which I will call the pseudoindex $\operatorname{PsI}(X)$ of $X$. In general it can be expected that $I(X) \neq P s I(X)$ (see [CTS], see also below).

A third invariant, related to $I(X)$ and $\operatorname{PSI}(X)$ is the period $P(X)$ of $X$. When $X$ is a principal homogeneous space for an abelian variety $A$ over a field $K$, this is the order of the class of $X$ in the group $H^{1}(K, A)$. For arbitrary smooth and proper $X$ over a field $K$, the period of $X$ is defined to be the period of the Albanese torsor $\operatorname{Alb}^{1}(X)$ associated to zerocycles of degree 1 , which is a a principal homogeneous space over the Albanese variety $\operatorname{Alb}(X)$. In other words, $P(X)$ is the order of the cokernel of the degree map

$$
\operatorname{Alb}^{*}(X)(k) \rightarrow \mathbf{Z}
$$


where $\operatorname{Alb}^{*}(X)$ is the total Albanese variety (see Section 1.1 for more details on the Albanese variety).

Lemma. Let $X$ be a smooth projective irreducible variety over an arbitrary field. We have

$$
P(X)|\operatorname{Ps} I(X)| I(X) .
$$

PROOF. The existence of a cycle map compatible with push-forward (see Section 3.1) implies that $P S I(X)$ divides $I(X)$. The results of Section 3.2 imply that we have a natural map ${ }^{1} H_{0}(X, \mathbf{Z}) \rightarrow \mathrm{Alb}^{*}(X)(k)$, compatible with push-forward, so $P(X)$ divides $P S I(X)$.

Corollary. Let $X$ be a principal homogeneous space for an abelian variety over a $p$-adic field $K$. The natural map

$$
\mathrm{Br}(K) \rightarrow \operatorname{Br}(K(X))
$$

from the Brauer group of $K$ to the Brauer group of the function field of $X$ is injective if and only if $X$ is a trivial principal homogeneous space.

Proof. Since $X$ is isomorphic to its Albanese torsor $\operatorname{Alb}^{1}(X)$, we have that $X$ is trivial if and only if $P(X)=1$. Hence, by Theorem 2 we want to show that $\operatorname{PsI}(X)=1$ if and only if $P(X)=1$. The 'only if' follows from the lemma, and the 'if' is obvious, since when $X$ is trivial, we have that $X(K) \neq \emptyset$, so $\operatorname{PsI}(X)=1$ by the covariant functoriality of pseudo-motivic homology.

\section{Remarks.}

(i) When $X$ is a principal homogeneous space for an abelian variety $A$ of dimension $d$ over an arbitrary field $K$ we also have an upper bound on $I(X)$ in terms of $P(X)$ :

$$
I(X) \mid P(X)^{2 d}
$$

as we can see from the map

$$
X \simeq \operatorname{Alb}^{1}(X) \stackrel{\times P(X)}{\longrightarrow} \operatorname{Alb}^{P(X)}(X) \simeq A
$$

which has degree $P(X)^{2 d}$.

(ii) For a smooth, proper variety $X$ over a $p$-adic field $K$ we actually have that $P(X)$ equals the order of the image of the map $\operatorname{Pic}^{0}(X) \rightarrow \operatorname{Br}(K)$ induced by the map $\operatorname{Pic}(X) \rightarrow$ $\operatorname{Br}(K)$ that appears in the Hochschild-Serre spectral sequence. In other words, $P(X)$ is equal to the order of the cokernel of the map from the group $\operatorname{Pic}^{0}(X)$ to the $K$-points of the Picard variety $\operatorname{Pic}^{0}(X / K)(K)$. This was already observed in [vH1, Rem. 5.4]. There I have given a sketch of a proof that follows Lichtenbaum's approach by using direct cocycle calculations. Section 4.3 contains a proof in the present framework.

\section{Zero-cycles and pseudo-motivic homology}

A different, less technical way to generalise Lichtenbaum's pairing to a higher dimensional smooth proper variety $X$ over a $p$-adic field $K$ is by a pairing

$$
\mathrm{CH}_{0}(X) \times \mathrm{Br}(X) \rightarrow \mathrm{Br}(K) .
$$

On the level of zero-cycles it is defined in exactly the same way as Lichtenbaum's original pairing (see for example [CTS]). It can be checked from the definitions that this pairing is compatible with our pairing ${ }^{1} H_{0}(X, \mathbf{Z}) \times \operatorname{Br}(X) \rightarrow \operatorname{Br}(K)$ via the cycle map

$$
\mathrm{cl}: C H_{0}(X) \rightarrow{ }^{1} H_{0}(X, \mathbf{Z})
$$


defined in Section 3.1. Generalised Lichtenbaum-Tate duality then implies that questions about the left and the right kernel of the pairing (4) are equivalent to questions about injectivity and surjectivity of the cycle map (5).

Since a $p$-adic field has infinite transcendence degree over $\mathbf{Q}$, the kernel of the cycle map (5) should in general contain a huge uniquely divisible subgroup, like it is the case over the complex numbers (D. Mumford's result, later extended by S. Bloch, see [B1]). Moreover, the examples of Parimala and Suresh in [PS] show that even for a conic bundle over a curve the kernel of the cycle map can contain torsion. This is unlike the situation over an algebraically closed field, where the kernel of the Albanese map is uniquely divisible by A. Rojtman's theorem (see [Ro]).

On the other hand, in [CTS] J.-L. Colliot-Thélène and S. Saito made the observation that $\mathrm{CH}_{0}(\mathrm{X})$ is orthogonal to the image of the Brauer group of a proper model $\mathfrak{X}$ over the ring of integers of $K$, which implies that the cycle map is not surjective if $\operatorname{Br}(\mathfrak{X}) \rightarrow \operatorname{Br}(X)$ is nonzero. Moreover, when $\mathfrak{X}$ is a regular proper model of $X$ over the integers of $K$, then the right kernel of (4) is exactly the image of $\operatorname{Br}(\mathfrak{X}) \rightarrow \operatorname{Br}(X)$. In particular, the index $I(X)$ of $X$ appears as the order of the kernel of the map $\operatorname{Br}(K) \rightarrow \operatorname{Br}(X) / \operatorname{Br}(\mathfrak{X})$. See [CTS, Th. 3.1] for the proof up to powers of $p$.

This suggests that a better approximation to $\mathrm{CH}_{0}(X)$ would be given by pseudo-motivic homology of a proper model $\psi: \mathfrak{X} \rightarrow \mathscr{O}_{K}$ over the ring of integers $\operatorname{Spec} \mathscr{O}_{K}$. Roughly speaking, this homology should be defined as

$$
{ }^{1} H_{*}\left(\mathfrak{X} / \mathscr{O}_{K}, \mathbf{Z}\right):=\operatorname{Ext}^{-*}\left(R \psi_{*} \mathbf{G}_{m}, \mathbf{G}_{m}\right) .
$$

However, to get groups with good properties, one should deal with the problems in characteristic $p$, even when $\mathfrak{X}$ has good reduction (compare Section 2.3). Moreover, to prove any kind of duality would be much harder, especially when $\mathfrak{X}$ has bad reduction.

\section{Pseudo-motivic homology over global fields}

We have seen that pseudo-motivic homology allows us to reinterpret pairings between zero-cycles and étale cohomology in terms of a cycles map. This makes pseudo-motivic homology a very convenient formalism to understand such pairings. A good example outside the topic of the present paper is the problem to understand the so-called reciprocity obstructions to the local-global principle for zero-cycles and rational points of varieties over global fields. In [vH2] pseudo-motivic homology and global duality have been used to reinterpret and refine results by E. Frossard concerning the Brauer-Manin obstruction to the localglobal principle for zero cycles on Severi-Brauer fibrations over curves over number fields. In [vH3] pseudo-motivic homology and global duality have been used to obtain results on the reciprocity obstruction to the Hasse principle for compactifications of torsors under tori over generalised global fields (such as function fields of curves over $p$-adic fields).

\section{Preliminaries}

In this section we will fix some notation and terminology and we will briefly consider the cohomology of sheaves on the smooth site over a scheme. The notation and terminology in this paper concerning derived categories is all standard, except maybe the choice not to make a distinction in terminology or notation between cohomology and 'hypercohomology' 
(the classical term for the result of applying a higher derived functor to a complex, rather than a single object). A sheaf will be a sheaf of abelian groups, unless mentioned otherwise.

A variety over a field $k$ will be a separated geometrically reduced (but not necessarily irreducible) scheme over $k$, and it will be of finite type unless mentioned otherwise (the group varieties we encounter will in general only be locally of finite type). When there is no danger of confusion, we will denote the scheme Speck by $k$. The base change of a variety $X$ over $k$ to an extension field $k^{\prime}$ will be denoted by $X_{k^{\prime}}$, and the base change to the separable closure $\bar{k}$ of $k$ will be denoted by $\bar{X}$. A curve over $k$ will be a variety over $k$ of pure dimension 1 .

\subsection{Picard and Albanese variety}

We will start by recalling some well-known results; the main reason for repeating them is to fix the notation and terminology, since there does not seem to be a well-established standard.

For a proper variety $\varphi: X \rightarrow k$ over a field $k$, the higher direct image sheaf $R^{1} \varphi_{*} \mathbf{G}_{m}$ on the $f p q c$-site over $k$ is represented by a group scheme locally of finite type over $k$ (see [Mur, II.15]), hence by a group variety locally of finite type if $k$ is of characteristic zero. In that case we will denote the group variety representing $R^{1} \varphi_{*} \mathbf{G}_{m}$ by $\operatorname{Pic}(X / k)$ and call it the total Picard variety of $X$. In general the Picard group $\operatorname{Pic}(X)=H^{1}\left(X, \mathbf{G}_{m}\right)\left(=H_{\mathrm{fpqc}}^{1}\left(X, \mathbf{G}_{m}\right)\right)$ does not coincide with the group of $k$-points of $\operatorname{Pic}(X / k)$ : we have the well-known exact sequence

$$
0 \rightarrow \operatorname{Pic}(X) \rightarrow \operatorname{Pic}(X / k)(k) \rightarrow \operatorname{Br}(k) \rightarrow \operatorname{Br}(X),
$$

where $\operatorname{Br}(X)$ denotes the cohomological Brauer group of $X$.

From now on we will assume that $X$ is smooth and proper over a field $k$ of characteristic zero. Then the connected component of $\operatorname{Pic}(X / k)$ containing zero is an abelian variety over $k$ which we denote by $\operatorname{Pic}^{0}(X / k)$ and which we call the Picard variety of $X$. We have an exact sequence

$$
0 \rightarrow \operatorname{Pic}^{0}(X / k) \rightarrow \operatorname{Pic}(X / k) \rightarrow N S(X / k) \rightarrow 0,
$$

where $N S(X / k)$ is the finitely generated group variety corresponding to the Néron-Severi group of $\bar{X}$, equipped with its natural Galois action. We denote by $\operatorname{Pic}^{0}(X)$ the inverse image of $\operatorname{Pic}^{0}(X / k)$ under the canonical injection $\operatorname{Pic}(X) \hookrightarrow \operatorname{Pic}(X / k)(k)$ and we put

$$
N S(X):=\operatorname{Pic}(X) / \operatorname{Pic}^{0}(X) .
$$

As is the case for Pic, we have that $N S(X) \neq N S(X / k)(k)$ in general.

In order to define the (total) Albanese variety, we consider the $f p q c$-sheaf $\mathbf{Z}^{X}$ on $k$ associated to the presheaf that sends a scheme $U$ to the free abelian group generated by the set $X(U)$ of maps from $U$ to $X$. Still assuming $X$ to be smooth and proper over a field $k$ of characteristic zero, we have that the sheaf $\mathbf{Z}^{X}$ admits a homomorphism

$$
\alpha: \mathbf{Z}^{X} \rightarrow \operatorname{Alb}^{*}(X)
$$

into a sheaf represented by a group variety locally of finite type over $k$ of which the connected component $\operatorname{Alb}(X)$ containing zero is an abelian variety. The map $\alpha$ is the universal homomorphism of $\mathbf{Z}^{X}$ to sheaves represented by group varieties of which the connected component containing zero is a (semi-)abelian variety (see for example [Ra, §1]). We will 
call $\operatorname{Alb}^{*}(X)$ the total Albanese variety of $X$. The abelian variety $\operatorname{Alb}(X)$ is the (classical) Albanese variety of $X$. When $X$ is geometrically irreducible, we have a short exact sequence

$$
0 \rightarrow \operatorname{Alb}(X) \rightarrow \operatorname{Alb}^{*}(X) \rightarrow \mathbf{Z} \rightarrow 0,
$$

where the map to $\mathbf{Z}$ corresponds, via $\alpha$, to the degree map $\mathbf{Z}^{X} \rightarrow \mathbf{Z}$. The connected component of $\mathrm{Alb}^{*}(X)$ mapping to $n \in \mathbf{Z}$ will be denoted by $\operatorname{Alb}^{n}(X)$. In particular, $\operatorname{Alb}^{0}(X)=\operatorname{Alb}(X)$, and $\alpha$ induces a morphism from $X$ to $\operatorname{Alb}^{1}(X)$ the Albanese torsor of $X$, which is a principal homogeneous space over $\operatorname{Alb}(X)$. Of course, any $k$-valued point $x \in X(k)$ induces, by subtraction, an isomorphism $\operatorname{Alb}^{1}(X) \rightarrow \operatorname{Alb}^{0}(X)$ of principal homogeneous spaces over $\operatorname{Alb}(X)$, hence a morphism $\alpha_{x}: X \rightarrow \operatorname{Alb}(X)$. This is the classical Albanese map for the pair $(X, x)$, which is universal for maps of $X$ into abelian varieties that send $x$ to zero. It is well-known that the Albanese variety and the Picard variety are each other's duals as abelian varieties. It is also an easy consequence of Theorem 3.6 below.

Remark 1.1. The terms 'Picard group', 'Picard variety' and 'Albanese variety' are traditional, and so is the notation $\operatorname{Pic}(X)$ for the Picard group and $\operatorname{Alb}(X)$ for the Albanese variety. The notation $\operatorname{Pic}(X / k)$ and $\operatorname{Alb}^{1}(X)$ is a variation on notation introduced by Grothendieck in [Gr1]. What I call here the 'total Picard variety' is often called the Picard scheme. By analogy, the term 'Albanese scheme' is used in [Ra] for what I call the 'total Albanese variety'. Indeed, when a variety is defined to be irreducible and/or of finite type, a distinguishing feature of the 'Picard scheme' and the 'Albanese scheme' is that they are not varieties. However, when varieties are not required to be irreducible, and when group varieties are allowed to be only locally of finite type, like in the present paper, this terminology does not make sense. Therefore, the adjective 'total' seems a better way to make the distinction.

\subsection{Smooth cohomology}

For a scheme $X$ the site $X_{\mathrm{sm}}$ has as underlying category the category of smooth schemes locally of finite type over $X$. The coverings are the smooth surjective morphisms. For us it will be more useful than more popular sites, like the (small) étale site $X_{\text {ét }}$ or the (big) flat site $X_{\mathrm{fl}}$, for which the underlying category consists of schemes that are étale and of finite type over $X$ (resp. locally of finite type over $X$ ) and the coverings are the surjective étale (resp. flat) morphisms. The reason is that, as we will see below, the underlying category is big enough to give reasonable Hom- and Ext-groups between smooth group schemes, but on the other hand small enough to compute the higher direct image sheaves $R \varphi_{*} \mathbf{G}_{m}$ we are interested in. The properties we use do not depend so much on the topology chosen. For example, everything in this paper would remain valid if we would replace every smooth site $X_{\mathrm{sm}}$ by the 'smooth-étale' site $X_{\mathrm{SmEt}}$, where the underlying category is still the category of smooth schemes locally of finite type over $X$, but the coverings are now the étale surjective morphisms.

\section{Comparison of topologies}

The cohomology of a sheaf $\mathscr{G}$ represented by a smooth commutative group scheme over $X$ is not very sensitive to the choice of sites: we have equalities

$$
H^{i}\left(X_{\mathrm{fl}}, \mathscr{G}\right)=H^{i}\left(X_{\mathrm{sm}}, \mathscr{G}\right)=H^{i}\left(X_{\text {ét }}, \mathscr{G}\right)
$$


for any $i$. This follows from the vanishing of higher direct images of the sheaf $\mathscr{G}$ for the mappings between the various topologies (see [Gr2, III, Th. 11.7], or [M1, Th. III.3.9]). Formula (8) also holds when $\mathscr{G}$ is a direct limit of sheaves represented by smooth group schemes, or when $\mathscr{G}$ is a complex of sheaves for which all cohomology sheaves $\mathscr{H}^{*}(\mathscr{G})$ are of the above form.

In the above situation we will often omit the reference to the topology and write $H^{i}(X, \mathscr{G})$ for any of the above groups. We will not make any distinction in notation between commutative group schemes and the sheaves they represent. Also, we will freely use the equivalence of categories between étale sheaves on $k$ and Galois modules. With these conventions, we have for example

$$
H^{i}\left(k_{\mathrm{sm}}, \mathbf{G}_{m}\right)=H^{i}\left(k_{\text {ét }}, \mathbf{G}_{m}\right)=H^{1}\left(k, \mathbf{G}_{m}\right)=H^{i}\left(k, \bar{k}^{*}\right)=H^{i}\left(\operatorname{Gal}(\bar{k} / k), \bar{k}^{*}\right) .
$$

With appropriate base change theorems we can also compare derived direct images. We will only use the first part of the following lemma. Part (ii) is included to show that for our purposes the only problem with the flat site is that we do not know whether the higher direct images $R^{q} \varphi_{*} \mathbf{G}_{m}$ are torsion for $q>1$ (see Section 2.2).

Lemma 1.2. Let $\varphi: X \rightarrow S$ be a morphism of finite type between schemes.

(i) Let $\mathscr{F}$ be an $n$-torsion sheaf on $X_{\mathrm{et}}$, where $n$ is invertible on $S$. Let $\alpha_{X}: X_{\mathrm{sm}} \rightarrow X_{\text {ét }}$ and $\alpha_{S}: S_{\mathrm{sm}} \rightarrow S_{\text {ét }}$ be the canonical morphisms of sites. Then the natural morphism

$$
\alpha_{S}^{*} R \varphi_{*} \mathscr{F} \rightarrow R \varphi_{*} \alpha_{X}^{*} \mathscr{F}
$$

is a quasi-isomorphism.

(ii) For $\alpha_{X}^{\prime}: X_{\mathrm{fl}} \rightarrow X_{\text {ét }}$ and $\alpha_{S}^{\prime}: S_{\mathrm{fl}} \rightarrow S_{\text {ét }}$ we also have an isomorphism $\alpha_{S}^{\prime *} R \varphi_{*} \mathscr{F} \rightarrow$ $R \varphi_{*} \alpha_{X}^{\prime *} \mathscr{F}$ if either:

(a) $\varphi$ is proper and $\mathscr{F}$ is a torsion sheaf on $X_{\text {ét }}$, or

(b) $S=$ Speck and $\mathscr{F}$ is a constructible $n$-torsion sheaf on $X_{\text {ét }}$ for $n$ prime to the characteristic of $k$,

PROOF. (i) This follows from the smooth base change theorem (see [SGA4, Cor. XVI.1.2] or [M1, Th. VI.4.1]) combined with the comparison between the smooth topology and the étale topology (8).

(ii) This follows from the proper base change theorem (see [SGA4, Th. XII.5.1] or [M1, Cor. VI.2.3]) in case (a), resp. Deligne's base change theorem [SGA4.5, Th. finitude, 1.9] in case (b), combined with the comparison between the flat topology and the étale topology (8).

\section{Comparison of Hom- and Ext- groups}

For our purposes here, the difference between the étale site and the smooth site lies in the internal and external Hom- and Ext-groups between sheaves represented by group schemes that are not locally constant. The étale site turns out to be too small to give good results; consider for example $\mathscr{H}_{o m_{\text {et }}}\left(\mathbf{G}_{m}, \mathbf{G}_{m}\right)=\operatorname{Hom}\left(\bar{k}^{*}, \bar{k}^{*}\right)$, which is not $\mathbf{Z}$ as it should be. Thanks to its bigger underlying category, the smooth site remembers the scheme structure of a smooth group scheme when we pass to the sheaf that it represents. The analogue is wellknown for the (big) flat site (see the references in the proof of Corollary 1.4 below), so here we only need to prove the following comparison result for internal derived homomorphisms. 
Lemma 1.3. Let $G_{1}, G_{2}$ be smooth group schemes locally of finite type over a scheme $X$. Let $\alpha: X_{\mathrm{fl}} \rightarrow X_{\mathrm{sm}}$ be the canonical morphism of sites. Then we have a canonical isomorphism

$$
R \alpha_{*} R \mathscr{H} o m_{X_{\mathrm{fl}}}\left(\mathscr{G}_{1}, \mathscr{G}_{2}\right)=R \mathscr{H} o m_{X_{\mathrm{sm}}}\left(\mathscr{G}_{1}, \mathscr{G}_{2}\right) .
$$

Proof. We have that $\alpha^{*}$ is exact (on the level of underlying categories $\alpha$ is a full embedding), so its right adjoint $\alpha_{*}$ sends injectives to injectives, and adjunction gives us an isomorphism

$$
R \alpha_{*} R \mathscr{H} O m_{X_{\mathrm{fl}}}\left(\alpha^{*} \mathscr{G}_{1}, \mathscr{G}_{2}\right)=R \mathscr{H} O m_{X_{\mathrm{sm}}}\left(\mathscr{G}_{1}, R \alpha_{*} \mathscr{G}_{2}\right) .
$$

Now $\alpha^{*} \mathscr{G}_{1}$ is represented by $\mathscr{G}_{1}$ on $X_{\mathrm{fl}}$, since $\mathscr{G}_{1}$ is a smooth group scheme. The complex $R \alpha_{*} \mathscr{G}_{2}$ is quasi-isomorphic to the sheaf represented by $\mathscr{G}_{2}$, since the higher direct images of $\mathscr{G}_{2}$ under $\alpha$ vanish, as we saw above.

Corollary 1.4. For $M$ a finitely generated group scheme, $T$ a torus, and $A$ an abelian variety over a field $k$ of characteristic zero we have

$$
\begin{aligned}
& R \mathscr{H} \mathrm{m}_{k_{\mathrm{sm}}}\left(M, \mathbf{G}_{m}\right)=\mathscr{H} \mathrm{m}_{\mathbf{G} / k}\left(M, \mathbf{G}_{m}\right), \\
& R \mathscr{H o m}_{\mathrm{sm}_{\mathrm{m}}}\left(T, \mathbf{G}_{m}\right)=\mathscr{H}_{\mathrm{om}} \mathbf{\mathrm { G } / k}\left(T, \mathbf{G}_{m}\right), \\
& R \mathscr{H} \operatorname{mom}_{k_{\mathrm{sm}}}\left(A, \mathbf{G}_{m}\right)=\mathscr{E} x t_{\mathbf{G} / k}^{1}\left(A, \mathbf{G}_{m}\right)[-1]=A^{t}[-1],
\end{aligned}
$$

where $\mathscr{H o m}_{\mathbf{G} / k}$ and $\mathscr{E} x t_{\mathbf{G} / k}^{1}$ are the internal Hom and Ext in the category $\mathbf{G} / k$ of commutative group schemes over $k$ and $A^{t}$ is the dual abelian variety of $A$.

Recall that the notation $[i]$ is used to indicate a shift by $i$ in the indexing of a complex. In particular, we have in the above corollary that $A^{t}[-1]$ denotes the complex consisting of the single object $A^{t}$ in degree 1 .

Proof. By Lemma 1.3 this follows from [O, Th. 17.4, Th. 18.1], [Br2, §§7, 8, 10], and the vanishing of the higher direct images under $\alpha: k_{\mathrm{fl}} \rightarrow k_{\mathrm{sm}}$ of sheaves represented by smooth group schemes.

\subsection{Motivic homology}

Apart from inspiring some terminology and notation, motivic (co)homology does not appear in the present paper. My grasp of that material would probably not have been sufficient to keep me out of trouble, and the results I wanted to prove here could all be obtained by classical techniques. For further applications of pseudo-motivic homology it might be desirable to extend the cycle map of Section 3.1 to a map $H_{i}(X, \mathbf{Z}) \rightarrow{ }^{1} H_{i}(X, \mathbf{Z})$ for all $i$, and also to a similar map from étale motivic homology. B. Kahn kindly explained to me that in the Suslin-Voevodsky framework this should be a more or less formal exercise, but I will not do it here.

\section{Pseudo-motivic homology}

Throughout this section, $\varphi: X \rightarrow k$ will be a smooth proper scheme over a field $k$ of characteristic zero. We will establish the basic properties of the pseudo-motivic homology groups

$$
{ }^{1} H_{*}(X, \mathbf{Z})=\operatorname{Ext}_{k_{\mathrm{sm}}}^{-*}\left(R \varphi_{*} \mathbf{G}_{m}, \mathbf{G}_{m}\right)
$$

and do some calculations. 
Observe that the definition of the pseudo-motivic homology groups ${ }^{1} H_{*}(X, \mathbf{Z})$ is completely analogous to the definitions à la Verdier of homology of locally compact spaces and of étale homology with finite coefficients:

(9) $H_{*}(X, \mathbf{Z} / n):=\operatorname{Ext}_{k_{\mathrm{et}}, \mathbf{Z} / n}^{-*}\left(R \varphi_{*} \mathbf{Z} / n, \mathbf{Z} / n\right)=\operatorname{Ext}_{k_{\mathrm{et}}, \mathbf{Z} / n}^{-*}\left(R \varphi_{*} \mu_{n}, \mu_{n}\right)=\operatorname{Ext}_{k_{\mathrm{et}}}^{-*}\left(R \varphi_{*} \mu_{n}, \mathbf{G}_{m}\right)$

(see for example [La], and recall that $X$ is proper over $k$ ). See Section 2.3 for further remarks on the definition and notation.

\subsection{Basic properties}

Kummer sequence

Applying the right derived functors of $\operatorname{Hom}_{k_{\mathrm{sm}}}\left(R \varphi_{*}-, \mathbf{G}_{m}\right)$ to the Kummer sequence

$$
0 \rightarrow \mu_{n} \rightarrow \mathbf{G}_{m} \rightarrow \mathbf{G}_{m} \rightarrow 0
$$

gives a long exact sequence

$$
\cdots \rightarrow{ }^{1} H_{i}(X, \mathbf{Z}) \stackrel{\times n}{\longrightarrow}{ }^{1} H_{i}(X, \mathbf{Z}) \rightarrow H_{i}(X, \mathbf{Z} / n) \rightarrow \cdots
$$

All basic constructions that follow below also exist for coefficients modulo $n$, and they are compatible with the Kummer exact sequences.

\section{Functoriality}

For a map $f: Y \rightarrow X$ of proper smooth schemes over $k$ the adjunction morphism $\mathbf{G}_{m} \rightarrow$ $R f_{*} \mathbf{G}_{m}$ induces the push-forward homomorphism $f_{*}:{ }^{1} H_{*}(Y, \mathbf{Z}) \rightarrow{ }^{1} H_{*}(X, \mathbf{Z})$. If $\pi: Y \rightarrow$ $X$ is finite étale, then the norm map $\pi_{*} \mathbf{G}_{m} \rightarrow \mathbf{G}_{m}$ (cf. [M1, Lemma V.1.12]) induces the étale pull-back $\pi^{*}:{ }^{1} H_{*}(X, \mathbf{Z}) \rightarrow{ }^{1} H_{*}(Y, \mathbf{Z})$. If $\pi$ is of constant degree $d$, then $\pi_{*} \circ \pi^{*}$ is multiplication by $d$. If $Y$ is Galois over $X$ with Galois group $G$, then then $\pi^{*} \circ \pi_{*}$ sends a class $\beta$ to the class $\sum_{g \in G} g \cdot \beta$.

Note that if $k^{\prime} / k$ is a finite extension and we have a base change diagram

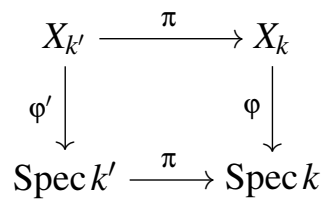

then the norm map induces an adjunction formula

$$
\operatorname{Ext}_{k_{\mathrm{sm}}^{\prime}}^{*}\left(R \varphi_{*}^{\prime} \mathbf{G}_{m}, \mathbf{G}_{m}\right)=\operatorname{Ext}_{k_{\mathrm{sm}}}^{*}\left(R\left(\pi \circ \varphi^{\prime}\right)_{*} \mathbf{G}_{m}, \mathbf{G}_{m}\right)
$$

(see [M1, Lemma V.1.12]). Therefore, the group ${ }^{1} H_{*}\left(X_{k^{\prime}}, \mathbf{Z}\right)$ does not depend on the question whether we consider $X_{k^{\prime}}$ as variety over $k$ or over $k^{\prime}$. In particular, we have a pushforward map $\pi_{*}$ and a pull-back map $\pi^{*}$ between the homology of $X_{k}$ and $X_{k^{\prime}}$.

Product with cohomology

The pairing (1) is a special case of the Yoneda pairing

$$
\begin{array}{rllc}
{ }^{1} H_{i}(X, \mathbf{Z}) & \times H^{j}\left(X, \mathbf{G}_{m}\right) & \rightarrow & H^{j-i}\left(k, \mathbf{G}_{m}\right) \\
\gamma & \times \omega & \mapsto & \gamma \cdot \omega
\end{array}
$$

which is defined for arbitrary $i, j$ via the canonical map

$$
\operatorname{Ext}_{k_{\mathrm{sm}}}^{-i}\left(R \varphi_{*} \mathbf{G}_{m}, \mathbf{G}_{m}\right)=\operatorname{Ext}_{k_{\mathrm{sm}}}^{0}\left(R \varphi_{*} \mathbf{G}_{m}, \mathbf{G}_{m}[-i]\right) \rightarrow \operatorname{Hom}\left(H^{j}\left(k, R \varphi_{*} \mathbf{G}_{m}\right), H^{j-i}\left(k, \mathbf{G}_{m}\right)\right) .
$$


From the definitions it is easy to check that for a morphism $f: Y \rightarrow X$ we have the projection formula

$$
f_{*} \gamma \cdot \omega=\gamma \cdot f^{*} \omega
$$

Homology of a point

For any finite field extension $k^{\prime}$ of $k$, we have a canonical isomorphism

$$
{ }^{1} H_{i}\left(\operatorname{Spec} k^{\prime}, \mathbf{Z}\right)=H^{-i}\left(k^{\prime}, \mathbf{Z}\right) .
$$

Under this isomorphism the push-forward morphism

$$
\pi_{*}:{ }^{1} H_{i}\left(\operatorname{Spec} k^{\prime}, \mathbf{Z}\right) \rightarrow{ }^{1} H_{i}(\operatorname{Spec} k, \mathbf{Z})
$$

corresponds to the norm map (i.e., the corestriction map in Galois cohomology). Moreover, the Yoneda product defined above corresponds for $X=\operatorname{Spec} k^{\prime}$ to the cup product

$$
H^{-i}\left(k^{\prime}, \mathbf{Z}\right) \times H^{j}\left(k^{\prime}, \mathbf{G}_{m}\right) \rightarrow H^{j-i}\left(k^{\prime}, \mathbf{G}_{m}\right)
$$

followed by the norm map

$$
H^{j-i}\left(k^{\prime}, \mathbf{G}_{m}\right) \rightarrow H^{j-i}\left(k, \mathbf{G}_{m}\right) .
$$

Remark 2.1. The above connection to Galois cohomology shows that the pseudo-motivic homology of Spec $k$ is in general not equal to the Galois homology group $H_{i}(\operatorname{Gal}(\bar{k} / k), \mathbf{Z})$, so we will avoid the notation ${ }^{1} H_{i}(k, \mathbf{Z})$, which might lead to misunderstandings.

\subsection{Calculations}

In this section $\varphi: X \rightarrow k$ will be a proper smooth geometrically irreducible variety of dimension $d$ over a field of characteristic zero. The condition of geometric irreducibility is merely for ease of exposition.

\section{A filtration on the derived direct image of $\mathbf{G}_{m}$}

In order to compute the pseudo-motivic homology groups, we will first define a convenient filtration on $R \varphi_{*} \mathbf{G}_{m}$. Since we work in a derived category, where the notion of 'subcomplex' does not make sense, this filtration will simply be a sequence of morphisms

$$
0=\mathscr{F}_{-1} \rightarrow \mathscr{F}_{0} \rightarrow \cdots \rightarrow \mathscr{F}_{2 d+1}=\mathscr{F}_{2 d+2}=\cdots=\mathscr{F}_{\infty}=R \varphi_{*} \mathbf{G}_{m} .
$$

For every $i \geq 0$ we define the ith graded piece $\mathscr{G}_{i}$ to be the mapping cone of the map $\mathscr{F}_{i-1} \rightarrow \mathscr{F}_{i}$, giving a triangle

$$
\mathscr{F}_{i-1} \rightarrow \mathscr{F}_{i} \rightarrow \mathscr{G}_{i} \rightarrow \mathscr{F}_{i}[1] .
$$

Our filtration will have the property that each graded piece consists of a nice sheaf concentrated in a single degree. Here a nice sheaf is either a torsion sheaf, or a locally constant finitely generated sheaf, or a sheaf represented by a connected group scheme. We construct it by taking the canonical filtration $\mathscr{F}_{\bullet}^{\text {can }}=\tau_{\leq i} R \varphi_{*} \mathbf{G}_{m}$, and put a further filtration on the graded piece of degree one, $R^{1} \varphi_{*} \mathbf{G}_{m}=\operatorname{Pic}(X / k)$, which is an extension of the finitely generated $N S(X / k)$ by the abelian variety $\operatorname{Pic}^{0}(X / k)$. To be precise, we take

$$
\mathscr{F}_{0}:=\tau_{\leq 0} R \varphi_{*} G=\varphi_{*} \mathbf{G}_{m}=\mathbf{G}_{m},
$$


for $\mathscr{F}_{1}$ we take the mapping cone of the canonical map $\tau_{\leq 1} R \varphi_{*} \mathbf{G}_{m} \rightarrow N S(X / k)$, with the degree shifted by one, so that we have a triangle

$$
\mathscr{F}_{1} \rightarrow \tau_{\leq 1} R \varphi_{*} \mathbf{G}_{m} \rightarrow N S(X / k) \rightarrow \mathscr{F}_{1}[1],
$$

and for $i \geq 2$ we put

$$
\mathscr{F}_{i}:=\tau_{\leq i-1} R \varphi_{*} \mathbf{G}_{m} .
$$

Using the standard notation of $\mathscr{G}[n]$ for a complex that consists of a sheaf concentrated in degree $-n$, we get

$$
\mathscr{G}_{i}= \begin{cases}\mathbf{G}_{m} & \text { if } i=0, \\ \operatorname{Pic}^{0}(X / k)[-1] & \text { if } i=1, \\ N S(X / k)[-1] & \text { if } i=2, \\ R^{i-1} \varphi_{*} \mathbf{G}_{m}[1-i] & \text { if } i \geq 3 .\end{cases}
$$

The sheaves $R^{q} \varphi_{*} \mathbf{G}_{m}$ are torsion for $q \geq 2$, since $H^{q}\left(X \times U, \mathbf{G}_{m}\right)$ is torsion for $q \geq 2$ and $U$ smooth over over $k$ by [Gr2, II, Prop. 1,4]. In other words, we have for $i \geq 3$ that

$$
\mathscr{G}_{i}=\underset{n}{\lim }\left({ }_{n} \mathscr{G}_{i}\right)
$$

where ${ }_{n} \mathscr{G}_{i}$ is the complex consisting of the $n$-torsion of the sheaf $R^{i-1} \varphi_{*} \mathbf{G}_{m}$ in degree $i-1$. It now follows from the Kummer sequence and Lemma 1.2 that the sheaf $R^{q} \varphi_{*} \mathbf{G}_{m}$ is isomorphic to the locally constant sheaf associated to the Galois module $H^{q}\left(\bar{X}, \mathbf{G}_{m}\right)$ for $q \geq 2$ and in fact to $H^{q}(\bar{X}, \mathbf{Q} / \mathbf{Z}(1))$ for $q>2$, where $\mathbf{Q} / \mathbf{Z}(1)=\lim _{\longrightarrow} \mu_{n}$. In other words,

$$
\mathscr{G}_{i}= \begin{cases}\operatorname{Br}(\bar{X})[-2] & \text { if } i=3, \\ H^{i-1}(\bar{X}, \mathbf{Q} / \mathbf{Z}(1))[i-1] & \text { if } i \geq 4 .\end{cases}
$$

In particular, we have by [SGA4, Exp. X, Cor. 4.3] (see also [M1, Th. VI.1.1]) that $\mathscr{G}_{i}=0$ for $i>2 d+1$, hence that $\mathscr{F}_{2 d+1}=\mathscr{F}_{\infty}$, as we claimed in the beginning. Below, we will also use the fact that by [SGA4.5, Th. finitude] (see also [M1, Th. VI.2.1]) we have that ${ }_{n} \mathscr{G}_{i}$ is finite for any $i>2$ and any $n \in \mathbf{N}$.

Remark 2.2. I do not know whether $R^{q} \varphi_{*} \mathbf{G}_{m}$ is torsion for $q \geq 2$ when taken on sites for which the underlying category contains singular schemes, like the flat site.

\section{The dual cofiltration}

For any complex $\mathscr{C}$ of sheaves on $k_{\text {sm }}$ we define the Cartier dual of $\mathscr{C}$ to be the complex

$$
\mathscr{C}^{D}:=R \mathscr{H}_{o m_{\mathrm{sm}}}\left(\mathscr{C}, \mathbf{G}_{m}\right) \text {. }
$$

In particular, ${ }^{1} H_{i}(X, \mathbf{Z})=H^{-1}\left(k_{\mathrm{sm}},\left(R \varphi_{*} \mathbf{G}_{m}\right)^{D}\right)$. Dualising the ascending filtration $\mathscr{F}$. on $R \varphi_{*} \mathbf{G}_{m}$ we get a descending cofiltration

$$
\left(R \varphi_{*} \mathbf{G}_{m}\right)^{D}=\mathscr{F}_{\infty}^{D}=\cdots=\mathscr{F}_{2 d+2}^{D}=\mathscr{F}_{2 d+1}^{D} \rightarrow \mathscr{F}_{2 d}^{D} \rightarrow \cdots \rightarrow \mathscr{F}_{0}^{D} \rightarrow \mathscr{F}_{-1}=\cdots=0,
$$

and for every $i \in \mathbf{Z}$ we have a triangle

$$
\mathscr{G}_{i}^{D} \rightarrow \mathscr{F}_{i}^{D} \rightarrow \mathscr{F}_{i-1}^{D} \rightarrow \mathscr{G}_{i}^{D}[1] .
$$

In order give explicit descriptions of the $\mathscr{G}_{i}^{D}$ we will first consider the case $i \geq 3$ in greater detail. Since the $\mathscr{G}_{i}$ are torsion for $i \geq 3$, we have that

$$
\mathscr{G}_{i}^{D}=R \mathscr{H} \operatorname{mom}_{k_{\mathrm{sm}}}\left(\underset{n}{\left(\lim _{n}\right.}\left(\mathscr{G}_{i}\right), \mathbf{G}_{m}\right)=R \underset{n}{\lim _{n}} R \mathscr{H}_{o m_{\mathrm{sm}}}\left({ }_{n} \mathscr{G}_{i}, \mathbf{G}_{m}\right) \text {. }
$$


For $i \geq 4$ the surjections $H^{i-1}\left(\bar{X}, \mu_{n}\right) \rightarrow{ }_{n} \mathscr{G}_{i}[i-1]$ and the isomorphisms $H_{i-1}(\bar{X}, \mathbf{Z} / n)=$ $R \mathscr{H} \operatorname{om}\left(H^{i-1}\left(\bar{X}, \mu_{n}\right), \mathbf{G}_{m}\right)$ induce an isomorphism

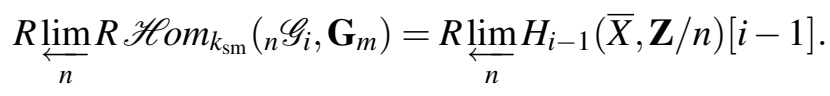

By [J, Th. 2.2] we have that

$$
H^{s}\left(k, \lim _{n} H_{t}(\bar{X}, \mathbf{Z} / n)\right)=H_{\text {cont }}^{s}\left(k, H_{t}(\bar{X}, \hat{\mathbf{Z}})\right),
$$

where $H_{\text {cont }}^{s}(k,-)$ denotes continuous Galois cohomology. Therefore we will write

$$
\mathscr{H}_{t}^{\text {cont }}(X / k, \hat{\mathbf{Z}}):=R{\underset{\leftarrow}{n}}_{\lim _{t}} H_{t}(\bar{X}, \mathbf{Z} / n) \text {. }
$$

Here we keep $k$ in the notation, since it is important that the inverse limit is taken in the derived category of sheaves on Spec $k$. For example, taking inverse limits does not commute with infinite field extensions (compare [K, §2]). In particular, when $k$ is not algebraically closed, the complexes $\mathscr{H}_{t}{ }^{\text {cont }}(X / k, \hat{\mathbf{Z}})$ will in general not be concentrated in degree 0 , whereas

$$
\mathscr{H}_{t}^{\text {cont }}(\bar{X} / \bar{k}, \hat{\mathbf{Z}})=R \underset{n}{\lim _{n}} H_{t}(\bar{X}, \mathbf{Z} / n)=H_{t}(\bar{X}, \hat{\mathbf{Z}})
$$

In the case $i=3$ we have that $\mathscr{G}_{3}^{D}$ equals the complex $R \mathscr{H} o m_{k_{\mathrm{sm}}}(\operatorname{Br}(\bar{X}), \mathbf{Q} / \mathbf{Z}(1))[2]$. As above, we have that

$$
H^{s}\left(k_{\mathrm{sm}}, \mathscr{G}_{3}^{D}\right)=H_{\text {cont }}^{s}(k, \operatorname{Hom}(\operatorname{Br}(\bar{X}), \mathbf{Q} / \mathbf{Z}(1)) .
$$

Combined with the above calculations of the $\mathscr{G}_{i}$ for $i=0,1,2$ and the results of Section 1, we get that

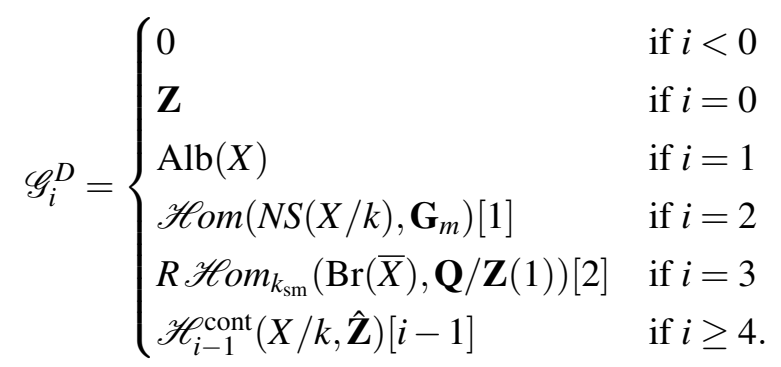

The modified Hochschild-Serre spectral sequence

Since the complexes $\mathscr{H}_{i-1}^{\text {cont }}(X / k, \hat{\mathbf{Z}})$ are in general not concentrated in degree 0 , the $E_{2}$-terms of the 'standard' Hochschild-Serre spectral sequence

$$
E_{2}^{s, t}=H^{s}\left(k_{\mathrm{sm}}, R^{t} \mathscr{H}_{0} m_{k_{\mathrm{sm}}}\left(R \varphi_{*} \mathbf{G}_{m}, \mathbf{G}_{m}\right)\right) \Rightarrow{ }^{1} H_{-s-t}(X, \mathbf{Z})
$$

do not admit a nice description in general. Therefore it makes sense to modify this spectral sequence a little, replacing the degree filtration on $\mathscr{H}_{o m_{\mathrm{sm}}}\left(R \varphi_{*} \mathbf{G}_{m}, \mathbf{G}_{m}\right)$, by the filtration

$$
0=\mathscr{F}_{-2 d-1}^{H S} \rightarrow \mathscr{F}_{-2 d}^{H S} \rightarrow \cdots \rightarrow \mathscr{F}_{0}^{H S}=R \mathscr{H}^{H S} m_{k_{\mathrm{sm}}}\left(R \varphi_{*} \mathbf{G}_{m}, \mathbf{G}_{m}\right)
$$

defined by

$$
\begin{aligned}
& \mathscr{F}_{i}^{H S}:=\left(\tau_{\geq-i} R \varphi_{*} \mathbf{G}_{m}\right)^{D} \text { if } i \neq-1, \\
& \mathscr{F}_{-1}^{H S}:=\text { mapping cone of }\left(R \varphi_{*} \mathbf{G}_{m}\right)^{D} \rightarrow \mathscr{F}_{1}^{D} .
\end{aligned}
$$


Here $\left(R \varphi_{*} \mathbf{G}_{m}\right)^{D} \rightarrow \mathscr{F}_{1}^{D}$ is the canonical map associated to the cofiltration $\mathscr{F}^{D}$ of $\left(R \varphi_{*} \mathbf{G}_{m}\right)^{D}$ defined above. Note that when $k$ is algebraically closed $\mathscr{F}^{H S}$ coincides with the degree filtration.

As above, we obtain for every $i$ the associated $i$ th graded piece $\mathscr{G}_{i}^{H S}$, and we put

$$
1_{\mathscr{H}_{i}}(X, \mathbf{Z}):=\mathscr{G}_{-i}^{H S}[-i] \text {. }
$$

The filtration $\mathscr{F}^{H S}$ gives rise to the modified Hochschild-Serre spectral sequence

$$
E_{2}^{s, t}=H^{s}\left(k_{\mathrm{sm}}, \mathscr{H}_{-t}(X, \mathbf{Z})\right) \Rightarrow{ }^{1} H_{-s-t}(X, \mathbf{Z}) .
$$

In this modified spectral sequence the $E_{2}$-terms are easy to interpret, thanks to the calculations above. For $i>0$ we have that

$$
{ }^{1} \mathscr{H}_{i}(X, \mathbf{Z})=\mathscr{G}_{i+1}^{D}[-i] .
$$

On the other hand, ${ }^{1} \mathscr{H}_{0}(X, \mathbf{Z})$ fits into an exact sequence

$$
0 \rightarrow \operatorname{Alb}(X) \rightarrow{ }^{1} \mathscr{H}_{0}(X, \mathbf{Z}) \rightarrow \mathbf{Z} \rightarrow 0 .
$$

This suggests that ${ }^{1} \mathscr{H}_{0}(X, \mathbf{Z})$ is represented by the total Albanese variety $\operatorname{Alb}^{*}(X)$ defined in Section 1.1. We will see in Section 3.2 that this is indeed the case. To simplify the notation, we will already use this fact below, but we will not allow ourselves to use the Albanese property of $\mathscr{H}_{0}(X, \mathbf{Z})$ in an essential way before Section 3.3.

In terms of Galois cohomology, we get the following expression for the $E_{2}$-terms of the modified Hochschild-Serre spectral sequence.

$$
H^{s}\left(k_{\mathrm{sm}}, \mathscr{H}_{-t}(X, \mathbf{Z})\right)= \begin{cases}0 & \text { if } t>0, \\ H^{s}\left(k, \operatorname{Alb}^{*}(X)(\bar{k})\right) & \text { if } t=0, \\ H^{s}\left(k, \operatorname{Hom}\left(N S(\bar{X}), \bar{k}^{*}\right)\right) & \text { if } t=-1, \\ H_{\mathrm{cont}}^{s}(k, \operatorname{Hom}(\operatorname{Br}(\bar{X}), \mathbf{Q} / \mathbf{Z}(1))) & \text { if } t=-2, \\ H_{\mathrm{cont}}^{s}\left(k, H_{-q}(\bar{X}, \hat{\mathbf{Z}})\right) & \text { if } t<-2 .\end{cases}
$$

Remark 2.3. If all Galois cohomology groups of $k$ with finite coefficient modules are finite, then

and

$$
H_{\text {cont }}^{s}(k, \operatorname{Hom}(\operatorname{Br}(\bar{X}), \mathbf{Q} / \mathbf{Z}(1)))={\underset{\leftarrow}{n}}_{\lim } H^{s}\left(k, \operatorname{Hom}\left({ }_{n} \operatorname{Br}(\bar{X}), \mathbf{Q} / \mathbf{Z}(1)\right)\right)
$$

$$
H_{\text {cont }}^{s}\left(k, H_{t}(\bar{X}, \hat{\mathbf{Z}})\right)=\underbrace{\lim }_{n} H^{s}\left(k, H_{t}(\bar{X}, \mathbf{Z} / n)\right)
$$

for any $s, t \in \mathbf{Z}$ (see [J, Rem. 3.5]). The finiteness condition is fulfilled when $k$ is a $p$-adic field.

\section{Calculations over an algebraically closed field}

For $\bar{X}$ over the algebraic closure $\bar{k}$ of $k$ the above gives us:

$$
{ }^{1} H_{i}(\bar{X}, \mathbf{Z})= \begin{cases}0 & \text { if } i<0, \\ \operatorname{Alb}^{*}(X)(\bar{k}) & \text { if } i=0, \\ \operatorname{Hom}\left(N S(\bar{X}), \bar{k}^{*}\right) & \text { if } i=1, \\ \operatorname{Hom}(\operatorname{Br}(\bar{X}), \mathbf{Q} / \mathbf{Z}(1)) & \text { if } i=2, \\ H_{i}(\bar{X}, \hat{\mathbf{Z}}) & \text { if } i>2 .\end{cases}
$$


High degree homology

Over an arbitrary field $k$ of characteristic 0 , we see from the modified Hochschild-Serre spectral sequence that the canonical map $R \varphi_{*} \mathbf{Q} / \mathbf{Z}(1) \rightarrow R \varphi_{*} \mathbf{G}_{m}$ induces for $i>2$ an isomorphism

$$
{ }^{1} H_{i}(X, \mathbf{Z})=R^{-i} \operatorname{Hom}_{k_{\mathrm{ett}}}\left(R \varphi_{*} \mathbf{Q} / \mathbf{Z}(1), \mathbf{G}_{m}\right) .
$$

The right hand side of this equation is canonically isomorphic to the $i$ th continuous étale homology group $H_{i}^{\text {cont }}(X, \hat{\mathbf{Z}})$ as defined in $[\mathrm{K}, \S 3.2]$ (recall that $X$ is proper over $k$ ).

\section{Calculations in degree 0 over a p-adic field}

Now let us assume $k$ has cohomological dimension $\leq 2$, which is the case when $X$ is a $p$-adic field (see [Se, Prop. II.15]). Then the $E_{2}^{s, t}$-terms of the modified Hochschild-Serre spectral sequence vanish for $s>2$. We get an exact sequence

$$
{ }^{1} H_{0}(X, \mathbf{Z}) \rightarrow \operatorname{Alb}^{*}(X)(k) \rightarrow H^{2}\left(k, \operatorname{Hom}\left(N S(\bar{X}), \bar{k}^{*}\right)\right) .
$$

The kernel ${ }^{1} H_{0}(X, \mathbf{Z}){ }^{\mathrm{Alb}}$ of the Albanese map ${ }^{1} H_{0}(X, \mathbf{Z}) \rightarrow \mathrm{Alb}^{*}(X)(k)$ fits into an exact sequence

$$
H_{\text {cont }}^{2}(k, \operatorname{Hom}(\operatorname{Br}(\bar{X}), \mathbf{Q} / \mathbf{Z}(1))) \rightarrow{ }^{1} H_{0}(X, \mathbf{Z})^{\mathrm{Alb}} \rightarrow H^{1}\left(k, \operatorname{Hom}\left(N S(\bar{X}), \bar{k}^{*}\right)\right) \rightarrow 0
$$

When $k$ is a $p$-adic field, we actually have

$$
H^{2}\left(k, \operatorname{Hom}\left(N S(\bar{X}), \bar{k}^{*}\right)\right)=\operatorname{Hom}\left(N S(\bar{X})^{\operatorname{Gal}(\bar{k} / k)}, \mathbf{Q} / \mathbf{Z}\right)
$$

and

$$
H_{\text {cont }}^{2}(k, \operatorname{Hom}(\operatorname{Br}(\bar{X}), \mathbf{Q} / \mathbf{Z}(1)))=\operatorname{Hom}\left(\operatorname{Br}(\bar{X})^{\operatorname{Gal}(\bar{k} / k)}, \mathbf{Q} / \mathbf{Z}\right)
$$

as we easily deduce from Tate duality for finitely generated groups (compare Proposition 4.1 .

\subsection{Further remarks on the definition}

\section{Notation}

As was mentioned in the introduction, I have chosen the notation ${ }^{1} H_{*}(X, \mathbf{Z})$ to distinguish pseudo-motivic homology from motivic homology. The ' 1 ' in the notation is to indicate that it is defined using the sheaf $\mathbf{G}_{m}$, which in the motivic setting is denoted by $\mathbf{Z}(1)[1]$. In fact, we can also define

$$
\begin{aligned}
{ }^{0} H_{i}(X, \mathbf{Z}) & :=\operatorname{Ext}_{k_{\mathrm{sm}}}^{-i}\left(R \varphi_{*} \mathbf{Z}, \mathbf{Z}\right), \\
{ }^{0} H_{i}(X, \mathbf{Z}(1)) & :=\operatorname{Ext}_{k_{\mathrm{sm}}}^{-i}\left(R \varphi_{*} \mathbf{Z}, \mathbf{G}_{m}[-1]\right) .
\end{aligned}
$$

These groups are almost to close to étale homology $H_{i}(X, \hat{\mathbf{Z}}(j))$ to be interesting. Still, they are a little bit nicer. At least we have ${ }^{0} H_{i}(\operatorname{Spec} k, \mathbf{Z})=\mathbf{Z}$, rather than $\hat{\mathbf{Z}}$, and for any smooth proper $X$ over a finite field we have that $C H_{0}(X) \simeq{ }^{0} H_{0}(X, \mathbf{Z})$.

I do not know whether there are complexes of sheaves $\mathbf{Z}(n)$ on $k_{\mathrm{sm}}$ that give reasonable groups ${ }^{n} H_{i}(X, \mathbf{Z}(j)):=\operatorname{Ext}_{k_{\mathrm{sm}}}^{-i}\left(R \varphi_{*} \mathbf{Z}(n), \mathbf{Z}(n+j)\right)$ for $n>1, j>-n$. In any case one would want ${ }^{n} H_{i}(\operatorname{Spec} k, \mathbf{Z}(j))=\mathbf{Z}(j)$ for any $j>-n$, and this already seems quite a strong condition. 
More general varieties or ground fields

Of course, we can consider the groups

$$
\operatorname{Ext}_{k_{\mathrm{sm}}}^{-i}\left(R \varphi_{*} \mathbf{G}_{m}, \mathbf{G}_{m}\right),
$$

for arbitrary $\varphi: X \rightarrow k$ over an arbitrary field $k$ (or even over an arbitrary base scheme). When $\varphi$ is smooth but not necessarily proper, and $k$ is a field of characteristic zero, this gives a nice homology theory with compact supports (see for example [vH2]).

In other cases these groups still have the same basic properties but as groups they might not be nice enough to deserve the name 'pseudo-motivic homology'. I should say that I do not know exactly what I mean by 'nice enough', but I would hope that at least we would have that the complex $R \mathscr{H} o m_{k_{\mathrm{sm}}}\left(R \varphi_{*} \mathbf{G}_{m}, \mathbf{G}_{m}\right)$ is concentrated in nonpositive degree, and that it admits a filtration for which the graded pieces are either complexes of group schemes, or profinite étale (compare Section 2.2). It is possible that even for singular varieties in characteristic zero the above definition is nice enough, but in general it will be hard to compute.

In characteristic $p>0$ the groups under consideration need not even be nice in the above sense when $X$ is smooth and proper, due to the 'pathological' behaviour of the $\mathscr{E} x t_{k_{\mathrm{sm}}}$ functor (see for example [Br1]). It is not clear to me whether this means that my notion of 'nice enough' is too restrictive, or that the definition should be adapted (by working over a different site, for example).

\section{The cycle map, the Albanese property and Poincaré duality}

In this section we will construct a cycle map for zero-cycles into the homology of degree zero, and check that this map satisfies the Albanese property. Then we discuss Deligne's result that implies Poincaré duality for curves.

\subsection{The cycle map for zero-cycles}

Let $k^{\prime}$ be a finite extension of a field $k$ of characteristic 0 . The canonical isomorphism (13) gives in degree zero a canonical isomorphism

$$
{ }^{1} H_{0}\left(\operatorname{Spec} k^{\prime}, \mathbf{Z}\right)=\mathbf{Z} .
$$

The canonical generator of ${ }^{1} H_{0}\left(\operatorname{Spec} k^{\prime}, \mathbf{Z}\right)$ can be called the fundamental class of Spec $k^{\prime}$. We will denote it by $\left[\operatorname{Spec} k^{\prime}\right] \in{ }^{1} H_{0}\left(\operatorname{Spec} k^{\prime}, \mathbf{Z}\right)$.

For a variety $X$ over $k$ we now define the cycle map

$$
\mathrm{cl}: \mathscr{Z}_{0}(X) \rightarrow{ }^{1} H_{0}(X, \mathbf{Z})
$$

from the group of zero-cycles into homology by sending a closed point $x \in X$ to the image of $[x]$ under the mapping $i_{*}:{ }^{1} H_{0}(x, \mathbf{Z}) \rightarrow{ }^{1} H_{0}(X, \mathbf{Z})$, where $i$ is the inclusion. By construction the cycle map commutes with the push-forward associated to a morphism of varieties $f: X \rightarrow Y$.

The following lemma implies that Lichtenbaum's pairing of $\mathscr{Z}_{0}(X)$ with $\operatorname{Br}(X)$, as defined in $[L 1, \S 3]$, factorises via the cycle map and the Yoneda pairing. 
Lemma 3.1. With notation as above, we have that for any $r \geq 0$ and any $\omega \in H^{r}\left(X, \mathbf{G}_{m}\right)$ the image of $\operatorname{cl}(x) \times \omega \in H^{r}\left(k, \mathbf{G}_{m}\right)$ under the pairing (11) coincides with the image of $\omega$ under the composite mapping

$$
H^{r}\left(X, \mathbf{G}_{m}\right) \stackrel{i^{*}}{\longrightarrow} H^{r}\left(x, \mathbf{G}_{m}\right) \stackrel{t r}{\longrightarrow} H^{r}\left(k, \mathbf{G}_{m}\right),
$$

where the mapping tr is induced by the norm map.

PROOF. Immediate from the definitions.

For the next result, it will be important that the cycle map for zero-cycles is already defined on the sheaf level. Let $\mathbf{Z}^{X}$ be the free sheaf on $k_{\mathrm{sm}}$ of abelian groups over $X$, i.e., the sheaf associated to the presheaf $U \mapsto \mathbf{Z}[X(U)]$. For every $U$ smooth over $k$ we have that a morphism $s: U \rightarrow X$ induces via pull-back a homomorphism from the complex of sheaves $R \varphi_{*} \mathbf{G}_{m}$ to the sheaf $\mathbf{G}_{m}$, both restricted to $U$. Thus we get a homomorphism

$$
c \ell: \mathbf{Z}^{X} \rightarrow R^{0} \mathscr{H} \circ \mathrm{om}\left(R \varphi_{*} \mathbf{G}_{m}, \mathbf{G}_{m}\right)={ }^{1} \mathscr{H}_{0}(X, \mathbf{Z})
$$

of sheaves on $k_{\mathrm{sm}}$. It follows from the definitions that the map

$$
\mathscr{Z}_{0}(X)=\mathbf{Z}^{X}(k) \stackrel{c \ell}{\longrightarrow} \mathscr{H}_{0}(X, \mathbf{Z})(k)
$$

obtained by taking sections over $k$ coincides with the composite map

$$
\mathscr{Z}_{0}(X) \stackrel{\mathrm{cl}}{\longrightarrow}{ }^{1} H_{0}(X, \mathbf{Z}) \rightarrow{ }^{1} \mathscr{H}_{0}(X, \mathbf{Z})(k) .
$$

In the next section we will see that $c \ell$ is actually the Albanese map into the total Albanese variety. In the result below it will be sufficient to know that $1 \mathscr{H}_{0}(X, \mathbf{Z})$ is represented by a commutative group variety locally of finite type, of which the connected component is an abelian variety. This implies that the cycle map $c \ell$ factorises via the total Albanese variety.

Proposition 3.2. Let $X$ be a proper smooth variety over a field of characteristic zero. The cycle map factorises via rational equivalence, giving a homomorphism

$$
\mathrm{cl}: \mathrm{CH}_{0}(X) \rightarrow{ }^{1} H_{0}(X, \mathbf{Z})
$$

Proof. Without loss of generality we may and will assume $X$ to be geometrically irreducible. The group $\mathscr{Z}_{0}^{\text {rat }}(X)$ of zero-cycles rationally equivalent to 0 is generated by zerocycles of the form $\pi_{*}(f)$, where $\pi: C \rightarrow X$ is a morphism of a nonsingular projective curve $C$ to $X$, and $(f)$ is the divisor of a rational function $f$ on $C$. Since $\operatorname{cl}\left(\pi_{*}(f)\right)=\pi_{*} \operatorname{cl}((f))$, it is sufficient to check the proposition for a nonsingular projective curve $C$.

The universal property of the total Albanese variety implies that the sheafified cycle map (20) factorises via the Albanese map $\alpha: \mathbf{Z}^{X} \rightarrow \operatorname{Alb}^{*}(X)$. Taking sections over $k$ we get a commutative diagram

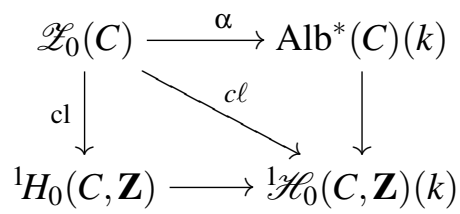

Since the map ${ }^{1} H_{0}(C, \mathbf{Z}) \rightarrow{ }^{1} \mathscr{H}_{0}(C, \mathbf{Z})(k)$ is injective by Hilbert's Theorem 90 , the kernel of the cycle map cl contains the kernel of the map $\alpha$, which is equal to $\mathscr{Z}_{0}^{\text {rat }}(C)$ by the Abel-Jacobi theorem. 


\subsection{The Albanese map}

In this section we will prove that the map

$$
c \ell: \mathbf{Z}^{X} \rightarrow \mathscr{H}_{0}(X, \mathbf{Z})
$$

defined above satisfies the Albanese property. In particular, ${ }^{1} \mathscr{H}_{0}(X, \mathbf{Z})$ is represented by the total Albanese variety $\operatorname{Alb}^{*}(X)$, as was claimed in Section 2.2.

The covariant functoriality of $\mathscr{H}_{0}(-, \mathbf{Z})$, will enable us to reduce the proof to the case where $X$ is a principal homogeneous space for an Abelian variety, and then the statement follows from the following result.

Proposition 3.3. Let $A$ be an abelian variety over a field $k$ of characteristic zero. The map

$$
\begin{aligned}
a: A & \rightarrow \mathscr{H}_{0}(A, \mathbf{Z})^{0} \\
x & \mapsto c \ell([x]-[0])
\end{aligned}
$$

is an isomorphism of (sheaves represented by) abelian varieties.

Remark 3.4. Since ${ }^{1} \mathscr{H}_{0}(A, \mathbf{Z})^{0}$ is the dual of $\operatorname{Pic}^{0}(A / k)$, as we saw in Section 2.2, it is well-known that $A$ is isomorphic to $\mathscr{H}_{0}(A, \mathbf{Z})^{0}$. There should be a way to compare the map $a$ directly with an existing construction of this isomorphism. However, I have not been able to find a suitable description in the literature that allowed me to make such a direct comparison, so I will make a detour via torsion coefficients. This is not quite satisfactory from a conceptual point of view, but we do get a very explicit relation to the Weil pairing as a bonus.

ProOf OF Proposition 3.3. The map $a$ is a priori only a morphism of varieties, but since ${ }^{1} \mathscr{H}_{0}(A, \mathbf{Z})^{0}$ is (represented by) an abelian variety, and 0 is mapped to 0 , it is a homomorphism of abelian varieties. In order to prove that $a$ is an isomorphism, it is sufficient to check that the induced map on $n$-torsion

$$
{ }_{n} A \rightarrow{ }_{n} 1 \mathscr{H}_{0}(A, \mathbf{Z})^{0}
$$

is an isomorphism for all $n \in \mathbf{N}$. This is equivalent to proving that the induced map of finite $n$-torsion groups

$$
a_{n}:{ }_{n} A(\bar{k}) \rightarrow{ }_{n}^{1} H_{0}(\bar{A}, \mathbf{Z})^{0}
$$

is an isomorphism. As we will see below, this follows from the nondegeneracy of the Weil pairing. We have that ${ }_{n}^{1} H_{0}(\bar{A}, \mathbf{Z})^{0}={ }_{n}^{1} H_{0}(\bar{A}, \mathbf{Z})=H_{1}(\bar{A}, \mathbf{Z} / n)=\operatorname{Hom}\left(H^{1}\left(\bar{A}, \mu_{n}\right), \bar{k}^{*}\right)=$ $\operatorname{Hom}\left({ }_{n} \operatorname{Pic}^{0}(\bar{A}), \bar{k}^{*}\right)$. Therefore we only have to check that the pairing between the $n$-torsion of $A(\bar{k})$ and $\operatorname{Pic}^{0}(\bar{A})$ induced by the map $a_{n}$ coincides up to sign with the Weil pairing. In the rest of the proof we will carefully deduce an explicit presentation of the pairing induced by $a_{n}$ in terms of functions and divisors. This presentation then turns out to coincide up to sign with a well-known presentation of the Weil pairing.

Let $\varphi: A \rightarrow k$ be the structure map, and let $\mathbf{n}: A \rightarrow A$ be multiplication by $n$. We define $R \varphi_{*} \mathbf{G}_{m} / \mathbf{n}^{*}$ to be the cone of the induced map

$$
R \varphi_{*} \mathbf{G}_{m} \stackrel{\mathbf{n}^{*}}{\longrightarrow} R \varphi_{*} \mathbf{G}_{m}
$$

and we put

$$
\begin{aligned}
H^{i}\left(\bar{A}, \mathbf{G}_{m}, \mathbf{n}^{*}\right) & :=H^{i}\left(\bar{k}, R \varphi_{*} \mathbf{G}_{m} / \mathbf{n}^{*}\right) \\
{ }^{1} H_{i}\left(\bar{A}, \mathbf{Z}, \mathbf{n}_{*}\right) & :=R^{-i} \operatorname{Hom}_{\bar{k}_{\mathrm{sm}}}\left(R \varphi_{*} \mathbf{G}_{m} / \mathbf{n}^{*}, \mathbf{G}_{m}\right) .
\end{aligned}
$$


We get long exact sequences

$$
H^{0}\left(\bar{A}, \mathbf{G}_{m}\right) \stackrel{\mathbf{n}^{*}}{\longrightarrow} H^{0}\left(\bar{A}, \mathbf{G}_{m}\right) \rightarrow H^{0}\left(\bar{A}, \mathbf{G}_{m}, \mathbf{n}^{*}\right) \rightarrow H^{1}\left(\bar{A}, \mathbf{G}_{m}\right) \stackrel{\mathbf{n}^{*}}{\longrightarrow} H^{1}\left(\bar{A}, \mathbf{G}_{m}\right) \rightarrow \cdots
$$

and

$$
\cdots \rightarrow{ }^{1} H_{1}(\bar{A}, \mathbf{Z}) \stackrel{\mathbf{n}_{*}}{\longrightarrow}{ }^{1} H_{1}(\bar{A}, \mathbf{Z}) \rightarrow{ }^{1} H_{0}\left(\bar{A}, \mathbf{Z}, \mathbf{n}_{*}\right) \rightarrow{ }^{1} H_{0}(\bar{A}, \mathbf{Z}) \stackrel{\mathbf{n}_{*}}{\longrightarrow}{ }^{1} H_{0}(\bar{A}, \mathbf{Z})
$$

Recall that the pull-back $\mathbf{n}^{*}$ is the identity on $\varphi_{*} \mathbf{G}_{m}$, multiplication by $n$ on $\operatorname{Pic}^{0}(A / k)$ and multiplication by $n^{2}$ on $N S(\bar{A})$. Also, recall that $N S(\bar{A})$ is torsion free (see [Mum, §8] for all these facts). This implies that

$$
H^{0}\left(\bar{A}, \mathbf{G}_{m}, \mathbf{n}^{*}\right)={ }_{n} \operatorname{Pic}(\bar{A})
$$

and

$$
{ }^{1} H_{0}\left(\bar{A}, \mathbf{Z}, \mathbf{n}_{*}\right)={ }_{n}^{1} H_{0}(\bar{A}, \mathbf{Z})
$$

It follows from the definition that $a$ induces a map

$$
a_{\mathbf{n}_{*}}:{ }_{n} A(\bar{k}) \rightarrow{ }^{1} H_{0}\left(\bar{A}, \mathbf{Z}, \mathbf{n}_{*}\right)
$$

that fits into the following commutative diagram.

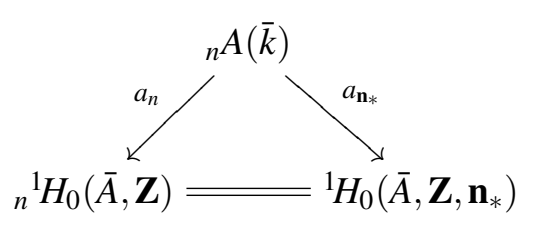

Hence it suffices to show that $a_{\mathbf{n}_{*}}$ is an isomorphism.

The cohomology sheaves $\mathscr{H}^{i}\left(R \varphi_{*} \mathbf{G}_{m} / \mathbf{n}^{*}\right)$ are torsion for every $i \in \mathbf{Z}$, as we see from the above expression of the endomorphism $\mathbf{n}^{*}$ as multiplication by powers of $n$. Therefore the comparison between smooth and étale cohomology gives us that the group ${ }^{1} H_{0}\left(\bar{A}, \mathbf{Z}, \mathbf{n}_{*}\right)$ is canonically isomorphic to the group $\operatorname{Hom}\left(R \varphi_{*} \mathbf{G}_{m} / \mathbf{n}^{*}, \bar{k}^{*}\right)$ computed in the derived category of étale sheaves on $\bar{k}$. We will now define a suitable complex of abelian groups that represents the complex of étale sheaves $R \varphi_{*} \mathbf{G}_{m} / \mathbf{n}^{*}$, in order to be able to compute $\operatorname{Hom}\left(R \varphi_{*} \mathbf{G}_{m} / \mathbf{n}^{*}, \bar{k}^{*}\right)$ explicitly.

Let $\mathscr{C}$ be the complex of abelian groups

$$
\mathscr{O}_{n}^{*} \bar{A}, \bar{A} \stackrel{\operatorname{div}}{\longrightarrow} \operatorname{Div}\left(\bar{A},{ }_{n} \bar{A}\right)
$$

where $\mathscr{O}_{n}^{*} \bar{A}, \bar{A}$ is the multiplicative group of invertible functions on $\bar{A}$ having no poles or zeroes on the $n$-torsion points, and $\operatorname{Div}\left(\bar{A},{ }_{n} \bar{A}\right)$ is the group of divisors on $\bar{A}$ with supports outside the $n$-torsion points. The moving lemma for divisors implies that $\mathscr{C}$ is quasiisomorphic to the complex

$$
\mathscr{K}(\bar{A})^{*} \stackrel{\operatorname{div}}{\longrightarrow} \operatorname{Div}(\bar{A}),
$$

hence we have a canonical map of complexes

$$
\mathscr{C} \rightarrow R \varphi_{*} \mathbf{G}_{m},
$$

that induces an isomorphism in cohomology of degree $\leq 1$. 


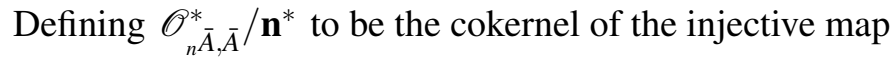

$$
\begin{aligned}
\mathscr{O}_{n}^{*}{ }_{n} \bar{A}, \bar{A} & \rightarrow \mathscr{O}^{*}{ }_{n} \bar{A}, \bar{A} \\
f & \mapsto f \circ \mathbf{n}
\end{aligned}
$$

and $\operatorname{Div}\left(\bar{A},{ }_{n} \bar{A}\right) / \mathbf{n}^{*}$ to be the cokernel of the injective map

$$
\begin{aligned}
\operatorname{Div}\left(\bar{A},{ }_{n} \bar{A}\right) & \rightarrow \operatorname{Div}\left(\bar{A},{ }_{n} \bar{A}\right) \\
D & \mapsto \mathbf{n}^{-1}(D),
\end{aligned}
$$

we see that the corresponding complex $\mathscr{C} / \mathbf{n}^{*}$ maps canonically to $R \varphi_{*} \mathbf{G}_{m} / \mathbf{n}^{*}$, inducing an isomorphism

$$
\begin{aligned}
H^{0}\left(\mathscr{C} / \mathbf{n}^{*}\right) \stackrel{\sim}{\rightarrow} H^{0}\left(\bar{A}, \mathbf{G}_{m}, \mathbf{n}^{*}\right) & ={ }_{n} \operatorname{Pic}(\bar{A}) \\
\left([f]: \operatorname{div}(f)=\mathbf{n}^{-1} D\right) & \mapsto[D]
\end{aligned}
$$

that sends the class of a function $f$ on $\bar{A}$ with $\operatorname{div}(f)=\mathbf{n}^{-1}(D)$ for some divisor $D$ to the $n$-torsion divisor class $[D] \in \operatorname{Pic}(\bar{A})$. Since $\bar{k}^{*}$ is a divisible group, hence injective, we also see that

$$
{ }^{1} H_{0}\left(\bar{A}, \mathbf{Z}, \mathbf{n}_{*}\right)=\operatorname{Hom}\left(H^{0}\left(R \varphi_{*} \mathbf{G}_{m} / \mathbf{n}^{*}\right), \bar{k}^{*}\right)=\operatorname{Hom}\left(H^{0}\left(\mathscr{C} / \mathbf{n}^{*}\right), \bar{k}^{*}\right) .
$$

In particular, we obtain a perfect pairing between ${ }^{1} H_{0}\left(\bar{A}, \mathbf{Z}, \mathbf{n}_{*}\right)$ and ${ }_{n} \operatorname{Pic}(\bar{A})$ into $\bar{k}^{*}$, and the map $a_{\mathbf{n}_{*}}$ induces a pairing

$$
{ }_{n} A(\bar{k}) \times{ }_{n} \operatorname{Pic}(\bar{A}) \rightarrow \bar{k}^{*} .
$$

From the above discussion and the definition of the cycle map we see that this pairing is given by the formula

$$
(x,[D]) \mapsto f(x) / f(0),
$$

where $D$ is a divisor with support outside the $n$-torsion points of $\bar{A}$ and $f$ is a function with $\operatorname{div}(f)=\mathbf{n}^{-1}(D)$. In other words, this pairing coincides up to sign with the Weil pairing, which is nondegenerate (see for example [Mum, §20]). We conclude that $a_{\mathbf{n}^{*}}$ is an isomorphism for every $n \in \mathbf{N}$.

Corollary 3.5. Let $A^{\bullet}$ be an extension of $\mathbf{Z}$ by an abelian variety $A^{0}$ over a field $k$ of characteristic zero. Let $A^{1}$ be the connected component of $A^{\bullet}$ mapping to $1 \in \mathbf{Z}$. We have an isomorphism of sheaves on $k_{\mathrm{sm}}$ represented by group varieties

$$
a^{\bullet}: A^{\bullet} \stackrel{\sim}{\rightarrow} \mathscr{H}_{0}\left(A^{1}, \mathbf{Z}\right)
$$

such that $a^{\bullet}$ restricted to $A^{1}$ is the canonical map

$$
A^{1} \rightarrow{ }^{1} \mathscr{H}_{0}\left(A^{1}, \mathbf{Z}\right)
$$

of sheaves of sets induced by the cycle map cl.

Proof. When $A^{\bullet}$ splits (i.e., $A^{\bullet}$ is isomorphic to $A^{0} \times \mathbf{Z}$ ), we extend the isomorphism $a: A^{0} \stackrel{\sim}{\rightarrow} \mathscr{H}^{1}\left(A^{0}, \mathbf{Z}\right)^{0}$ defined above to an isomorphism $A^{\bullet} \stackrel{\sim}{\rightarrow} 1 \mathscr{H}_{0}\left(A^{1}, \mathbf{Z}\right)$ by sending $(x, i) \in$ $A^{0} \times \mathbf{Z}$ to $c \ell([(x, 1)]+(i-1)[(0,1)])$. For any $(y, 1) \in A^{1}(k)$ we have that $c \ell([(x-(i-$ 1) $y, 1)]+(i-1)[(y, 1)])=c \ell([(x, 1)]+i[(0,1)])$, so a different splitting $\mathbf{Z} \rightarrow A^{\bullet}$, mapping 1 to $(y, 1)$, induces the same isomorphism.

We now generalise this to arbitrary $A^{\bullet}$. For $T$ smooth over $k$ with $x_{1} \in A^{1}(T) \neq \emptyset$ we send $x \in A^{i}(T)$ to

$$
c \ell\left(\left[x-(i-1) x_{1}\right]+(i-1)\left[x_{1}\right]\right) \in{ }^{1} \mathscr{H}_{0}\left(A^{1}, \mathbf{Z}\right)(T),
$$


As above, we have that this map is independent of the choice of $x_{1}$. Since the extension

$$
0 \rightarrow A^{0} \rightarrow A^{\bullet} \rightarrow \mathbf{Z} \rightarrow 0
$$

is locally trivial on the smooth site over $k$, the above construction gives us an isomorphism $a^{\bullet}: A^{\bullet} \stackrel{\sim}{\rightarrow} \mathscr{H}_{0}\left(A^{1}, \mathbf{Z}\right)$.

Theorem 3.6. Let $X$ be a proper smooth geometrically irreducible variety over a field $k$ of characteristic zero. The homomorphism of sheaves

$$
c \ell: \mathbf{Z}^{X} \rightarrow{ }^{1} \mathscr{H}_{0}(X, \mathbf{Z})
$$

is the universal homomorphism of $\mathbf{Z}^{X}$ into sheaves on $k_{\mathrm{sm}}$ represented by group varieties locally of finite type of which the connected component containing zero is an abelian variety. In particular, ${ }^{1} \mathscr{H}_{0}(X, Z)$ is represented by the total Albanese variety of $X$.

ProOF. Let $A$ be a commutative group variety locally of finite type of which the connected component containing zero is an abelian variety. Let

$$
f: \mathbf{Z}^{X} \rightarrow A
$$

be a homomorphism of sheaves. We will show that $f$ factorises via the cycle map $c \ell$.

Let $A^{0}$ be the connected component of $A$ containing zero. In order to be able to use Corollary 3.5, we need to replace $A$ by an extension $A^{\bullet}$ of $\mathbf{Z}$ by $A^{0}$. Let $\mathbf{Z}_{0}^{X}$ be the subsheaf of $\mathbf{Z}^{X}$ of elements of degree zero. Since $X$ is geometrically connected, we have that $\mathbf{Z}_{0}^{X}$ maps to $A^{0}$, so $\mathbf{Z}^{X} / \mathbf{Z}_{0}^{X}(=\mathbf{Z})$ maps to $A / A^{0}$. We take the fibre product $A^{\bullet}=A \times_{A / A^{0}} \mathbf{Z}$, and we have a homomorphism

$$
f^{\bullet}: \mathbf{Z}^{X} \rightarrow A^{\bullet}
$$

defined by $f^{\bullet}(z)=(f(z), \operatorname{deg}(z))$. We denote by $\pi: A^{\bullet} \rightarrow A$ the canonical projection. In order to prove the theorem, it is sufficient to show that $f^{\bullet}$ factorises via the cycle map $c \ell$ and a homomorphism from ${ }^{1} \mathscr{H}_{0}(X, \mathbf{Z})$ to $A^{\bullet}$, since $f=f^{\bullet} \circ \pi$.

Let $A^{1} \subset A^{\bullet}$ be the connected component mapping to $1 \in \mathbf{Z}$, and let

$$
f^{1}: X \rightarrow A^{1}
$$

be the morphism of varieties induced by $f^{\bullet}$. By Corollary 3.5 we have the following commutative diagram.

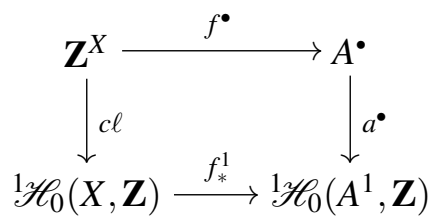

Since $a^{\bullet}$ is an isomorphism, the diagram gives the desired factorisation of $f^{\bullet}$ via ${ }^{1} \mathscr{H}_{0}(X, \mathbf{Z})$.

\subsection{Poincaré duality for curves}

In [SGA4, XVIII.1] P. Deligne (inspired by A. Grothendieck and J.-P. Serre) has constructed an isomorphism of $f p p f$-sheaves

$$
\tau_{\leq 0} R \varphi_{*} \varphi^{*} G[1] \stackrel{\sim}{\rightarrow} \tau_{\leq 0} R \mathscr{H} O{ } m_{S_{f p p f}}\left(\tau_{\leq 0} R \varphi_{*} \mathbf{G}_{m}[1], G[1]\right)
$$


for any smooth projective curve $\varphi: C \rightarrow S$ over a base scheme $S$, and any suitable (see [SGA4, XVIII.1.3.1.1]) fppf-sheaf $G$ on $S$. The sheaf $G$ can for example be a sheaf represented by a smooth group scheme of finite presentation. For $G=\mathbf{G}_{m}$ we get an isomorphism

$$
\tau_{\leq 1} R \varphi_{*} \mathbf{G}_{m} \stackrel{\sim}{\rightarrow} \tau_{\leq 1} R \mathscr{H} o m_{S_{f p p f}}\left(\tau_{\leq 1} R \varphi_{*} \mathbf{G}_{m}, \mathbf{G}_{m}[-1]\right)
$$

if we shift the degrees by 1 .

From Section 1.2 and the calculations in Section 2.2 we see that the isomorphism (22) induces an analogous isomorphism on the smooth site over $S$, and that for that isomorphism all truncations $\tau_{\leq 1}$ are superfluous if $S$ is the spectrum of a field of characteristic zero. Hence for a curve $\varphi: C \rightarrow k$ over a field of characteristic zero we have that

$$
R \varphi_{*} \mathbf{G}_{m} \stackrel{\sim}{\rightarrow} R \mathscr{H}_{0 m_{\mathrm{sm}}}\left(R \varphi_{*} \mathbf{G}_{m}, \mathbf{G}_{m}[-1]\right) .
$$

By taking cohomology $H^{i}(k,-)$, we get for every $i \in \mathbf{Z}$ the Poincaré duality isomorphism

$$
H^{i}\left(C, \mathbf{G}_{m}\right) \simeq{ }^{1} H_{1-i}(C, \mathbf{Z})
$$

given in the introduction.

The Poincaré duality pairing

Note that the map (23) induces a pairing in the derived category of sheaves on $k_{\mathrm{sm}}$

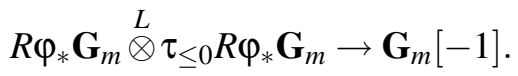

This gives for any $s, t \in \mathbf{Z}$ a pairing

$$
H^{s}\left(C, \mathbf{G}_{m}\right) \otimes H^{t}\left(C, \mathbf{G}_{m}\right) \rightarrow H^{s+t-1}\left(k, \mathbf{G}_{m}\right)
$$

which is compatible with the Yoneda pairing (11) and the Poincaré duality isomorphism (24).

We get more information on this pairing by looking at the results of [SGA4, XVIII.1.3.11-16], where the construction underlying the isomorphism (21) is studied in greater detail for $G=\mathbf{G}_{m}$. The following theorem summarises these results. In order to preserve the original generality, they are stated in terms of the pairing on the fppf-site induced by the map (22), rather than the pairing (25) on the smooth site.

Theorem 3.7 (P. Deligne (A. Grothendieck, J.-P. Serre), [SGA4, XVIII.1]). Let $\varphi: C \rightarrow S$ be a smooth projective curve over a scheme $S$. We have a symmetric pairing in the in the derived category of sheaves on $S_{f p p f}$

$$
\tau_{\leq 1} R \varphi_{*} \mathbf{G}_{m} \stackrel{L}{\otimes} \tau_{\leq 1} R \varphi_{*} \mathbf{G}_{m} \rightarrow \mathbf{G}_{m}[-1] .
$$

inducing an isomorphism

$$
\tau_{\leq 1} R \varphi_{*} \mathbf{G}_{m} \simeq \tau_{\leq 1} R \mathscr{H} O m_{S_{f p p f}}\left(\tau_{\leq 1} R \varphi_{*} \mathbf{G}_{m}, \mathbf{G}_{m}[-1]\right)
$$

such that

(i) The composite mapping

$$
\mathbf{Z}^{C} \stackrel{c \ell}{\longrightarrow} R^{0} \mathscr{H}_{0 m_{S f p p f}}\left(\tau_{\leq 1} R \varphi_{*} \mathbf{G}_{m}, \mathbf{G}_{m}[-1]\right) \simeq R^{1} \varphi_{*} \mathbf{G}_{m}=\operatorname{Pic}(C / S)
$$

(obtained from the above isomorphism and the cycle map (20)) coincides with the mapping $\mathbf{Z}^{C} \rightarrow \operatorname{Pic}(C / S)$ given on the presheaf level by sending a local section s to the isomorphism class of the invertible coherent sheaf $\mathscr{O}(s)$ associated to the image of $s$. 
(ii) We have a diagram of compatible pairings

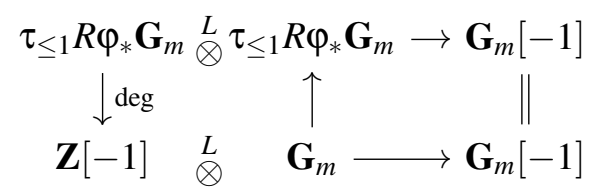

Here the arrow $\mathbf{Z}[-1] \otimes{ }^{L} \mathbf{G}_{m} \rightarrow \mathbf{G}_{m}[-1]$ is the canonical isomorphism, and $\mathbf{G}_{m} \rightarrow$ $R \varphi_{*} \mathbf{G}_{m}=R \varphi_{*} \varphi^{*} \mathbf{G}_{m}$ is the adjunction map (which induces the pull-back $\varphi^{*}: H^{i}\left(S, \mathbf{G}_{m}\right) \rightarrow$ $\left.H^{i}\left(C, \mathbf{G}_{m}\right)\right)$.

(iii) For a finite flat morphism of smooth projective curves over $S$

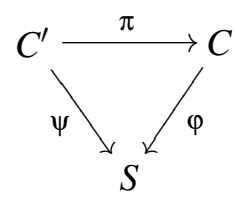

we have a diagram of compatible pairings

$$
\begin{aligned}
& \tau_{\leq 1} R \varphi_{*} \mathbf{G}_{m} \stackrel{L}{\otimes} \tau_{\leq 1} R \varphi_{*} \mathbf{G}_{m} \rightarrow \mathbf{G}_{m}[-1] \\
& \downarrow \pi^{*} \quad \uparrow_{N_{C^{\prime} / C}} \quad \| \\
& \tau_{\leq 1} R \psi_{*} \mathbf{G}_{m} \stackrel{L}{\otimes} \tau_{\leq 1} R \psi_{*} \mathbf{G}_{m} \rightarrow \mathbf{G}_{m}[-1]
\end{aligned}
$$

Here $N_{C^{\prime} / C}$ is the map induced by the norm map $\pi_{*} \mathbf{G}_{m} \rightarrow \mathbf{G}_{m}$.

Proof. We already saw that the perfectness of the pairing is a special case of [SGA4, XVIII.1.5.1], which is a reinterpretation of [SGA4, XVIII.1.3.10]. The other statements are reinterpretations of 1.3.16.2 (symmetry), 1.3.16.1 (compatibility (i)), 1.3.16.3 (compatibility (ii)), and 1.3.16.5 (compatibility (iii)) in loc. cit.

Remark. In view of the calculations of the pseudo-motivic homology groups in high degree (see Section 2.2), I do not expect that the above Poincaré duality generalises to higher dimensions. To be precise, I do not think that for $d>1$ there are (complexes of) sheaves ' $\mathbf{Z}(d)$ on the smooth site over $\mathbf{Q}$ such that for each proper smooth purely $d$-dimensional variety $X$ over a field of characteristic zero we have $H^{i}\left(X,{ }^{\prime} \mathbf{Z}(d)\right)={ }^{1} H_{2 d-i}(X, \mathbf{Z})$.

\section{Generalised Tate duality}

In this section we will prove that the complexes $\mathscr{F}_{i}$ defined in Section 2.2 satisfy Tate duality when $X$ is smooth and proper over a $p$-adic field. In particular, taking $i=\infty$ gives us Theorem 1. We derive Tate duality for the $\mathscr{F}_{i}$ from the classical Tate duality for the graded pieces $\mathscr{G}_{i}$ which are (direct limits of) étale finitely generated groups, tori or abelian varieties concentrated in a single degree (see Section 4.1).

A crucial role in the proof of Theorem 4.3 below will be played by the following collection (indexed by $i \geq 0$ ) of pairings of long exact sequences into $H^{2}\left(K, \mathbf{G}_{m}\right)=\mathbf{Q} / \mathbf{Z}$ :

$$
\begin{aligned}
\cdots & \leftarrow H^{r+1}\left(K, \mathscr{G}_{i}^{D}\right) \longleftarrow H^{r}\left(K, \mathscr{F}_{i-1}^{D}\right) \longleftarrow H^{r}\left(K, \mathscr{F}_{i}^{D}\right) \longleftarrow H^{r}\left(K, \mathscr{G}_{i}^{D}\right) \longleftarrow \cdots \\
\times \quad \times \quad \times & \times \\
\cdots & \rightarrow H^{1-r}\left(K, \mathscr{G}_{i}\right) \longrightarrow H^{2-r}\left(K, \mathscr{F}_{i-1}\right) \rightarrow H^{2-r}\left(K, \mathscr{F}_{i}\right) \rightarrow H^{2-r}\left(K, \mathscr{G}_{i}\right) \rightarrow \cdots
\end{aligned}
$$


For $i \geq 0$ this system is constructed from the triangles (14) and (16) using the Yoneda pairing. It will allow us to glue the Tate duality for the $\mathscr{G}_{i}$ (see Proposition 4.1) in order to obtain duality for the $\mathscr{F}_{i}$. This is done in Section 4.2. In the gluing process some caution is necessary, since some of the duality pairings for the $\mathscr{G}_{i}$ are only perfect after taking suitable completions. This means that we need to complete some of the groups in the long exact sequences (26), but without destroying the exactness. To identify these trouble spots we need the explicit calculations of Section 2.2. Then Lemma 4.2 provides the essential arguments that will allow us to proceed. Finally, Section 4.3 contains an application of Theorem 4.3 already announced in the introduction.

Throughout this section $X$ is a smooth, proper and geometrically irreducible variety over a $p$-adic field $K$.

\subsection{Classical Tate duality}

Recall from Section 2.2 that the graded pieces of the filtration $\mathscr{F}_{i}$ and its dual are as follows.

\begin{tabular}{|c|c|c|}
\hline$i$ & $\mathscr{G}_{i}$ & $\mathscr{G}_{i} D$ \\
\hline 0 & $\mathbf{G}_{m}$ & $\mathbf{Z}$ \\
\hline 1 & $\operatorname{Pic}^{0}(X / K)[-1]$ & $\operatorname{Alb}^{0}(X)$ \\
\hline 2 & $N S(X / K)[-1]$ & $\mathscr{H} \mathrm{m}_{k_{\mathrm{sm}}}\left(N S(X / K), \mathbf{G}_{m}\right)[1]$ \\
\hline 3 & $\operatorname{Br}(\bar{X})[-2]$ & $R \mathscr{H}{ }^{\prime} m_{k_{\mathrm{sm}}}\left(\operatorname{Br}(\bar{X}), \mathbf{G}_{m}\right)[2]$ \\
\hline 4 & $H^{3}(\bar{X}, \mathbf{Q} / \mathbf{Z}(1))[-3]$ & $\mathscr{H}_{3}^{\text {cont }}(X / k, \hat{\mathbf{Z}})[3]$ \\
\hline 5 & $H^{4}(\bar{X}, \mathbf{Q} / \mathbf{Z}(1))[-4]$ & $\mathscr{H}_{4}^{\text {cont }}(X / k, \hat{\mathbf{Z}})[4]$ \\
\hline$\vdots$ & $\vdots$ & $\vdots$ \\
\hline
\end{tabular}

Proposition 4.1. Let $X$ be a smooth and proper geometrically irreducible variety over a $p$-adic field $K$. Consider for $i \geq 0, r \in \mathbf{Z}$ the Yoneda pairing

$$
H^{r}\left(K, \mathscr{G}_{i}^{D}\right) \times H^{2-r}\left(K, \mathscr{G}_{i}\right) \rightarrow H^{2}\left(K, \mathbf{G}_{m}\right)=\mathbf{Q} / \mathbf{Z}
$$

(i) For every $i \geq 0, r \in \mathbf{Z}$ the pairing is nondegenerate.

(ii) For $i>2, r \in \mathbf{Z}$ the pairing is a perfect pairing between a profinite group and a torsion group.

(iii) For $i>2$ the groups in the pairing vanish if $r$ is not in the range $\{i-1, i+1\}$.

(iv) The pairing induces perfect pairings

$$
\begin{aligned}
& H^{2}\left(K, \mathscr{G}_{0}^{D}\right) \times H^{0}\left(K, \mathscr{G}_{0}\right)^{\wedge} \rightarrow \mathbf{Q} / \mathbf{Z}, \\
& H^{1}\left(K, \mathscr{G}_{1}^{D}\right) \times H^{1}\left(K, \mathscr{G}_{1}\right) \rightarrow \mathbf{Q} / \mathbf{Z}, \\
& H^{1}\left(K, \mathscr{G}_{2}^{D}\right) \times H^{1}\left(K, \mathscr{G}_{2}\right)^{\wedge} \rightarrow \mathbf{Q} / \mathbf{Z},
\end{aligned}
$$

between a torsion group and a profinite group, perfect pairings

$$
\begin{aligned}
H^{0}\left(K, \mathscr{G}_{0}^{D}\right)^{\wedge} \times H^{2}\left(K, \mathscr{G}_{0}\right) & \rightarrow \mathbf{Q} / \mathbf{Z}, \\
H^{0}\left(K, \mathscr{G}_{1}^{D}\right) \times H^{2}\left(K, \mathscr{G}_{0}\right) & \rightarrow \mathbf{Q} / \mathbf{Z}, \\
H^{-1}\left(K, \mathscr{G}_{2}^{D}\right)^{\wedge} \times H^{3}\left(K, \mathscr{G}_{2}\right) & \rightarrow \mathbf{Q} / \mathbf{Z}
\end{aligned}
$$


between a profinite group and a torsion group, and a perfect pairing

$$
H^{0}\left(K, \mathscr{G}_{2}^{D}\right) \times H^{2}\left(K, \mathscr{G}_{2}\right) \rightarrow \mathbf{Q} / \mathbf{Z}
$$

between finite groups.

(v) For $i=0$ or $1, r \in \mathbf{Z}$ the groups $H^{r}\left(K, \mathscr{G}_{i}^{D}\right)$ and $H^{2-r}\left(K, \mathscr{G}_{i}\right)$ vanish if the pair $(i, r)$ is not in the set $\{(0,0),(0,2),(1,0),(1,1)\}$. For $i>1$ the groups $H^{r}\left(K, \mathscr{G}_{i}^{D}\right)$ and $H^{2-r}\left(K, \mathscr{G}_{i}\right)$ vanish if $r$ is not in the range $1-i, \ldots, 3-i$.

ProOF. For $i=0,2$ the proposition follows from Hilbert's Theorem 90 and Tate duality for finitely generated groups (see [M2, Thm. I.2.1]). For $i=1$ the proposition follows from Tate duality for abelian varieties (see [M2, Cor. 3.4]).

For $i>2$ the proposition follows from Tate duality for finite groups, since

$$
\mathscr{G}_{i}=\underset{n}{\lim _{n}} \mathscr{G}_{i}
$$

where the complex ${ }_{n} \mathscr{G}_{i}$ consists of the (finite) $n$-torsion subgroup ${ }_{n} \mathscr{H}^{i-1}\left(\mathscr{G}_{i}\right)$ of the Galois module $\mathscr{H}^{i-1}\left(\mathscr{G}_{i}\right)=H^{i-1}\left(\bar{X}, \mathbf{G}_{m}\right)$ placed in degree $i-1$. Hence for all $r \in \mathbf{Z}$ we have

$$
H^{2-r}\left(K, \mathscr{G}_{i}\right)=\underset{n}{\lim } H^{2-r}\left(K,{ }_{n} \mathscr{G}_{i}\right),
$$

and

$$
H^{r}\left(K, \mathscr{G}_{i}^{D}\right)=H^{r}\left(K, R{\underset{n}{n}}_{\lim _{n}}\left(\mathscr{G}_{i}\right)^{D}\right)=R{\underset{n}{\lim _{n}}}^{r} H^{r}\left(K,\left({ }_{n} \mathscr{G}_{i}\right)^{D}\right)={\underset{\varliminf}{n}}_{\lim } H^{r}\left(K,\left({ }_{n} \mathscr{G}_{i}\right)^{D}\right),
$$

since the groups $H^{r}\left(K,\left({ }_{n} \mathscr{G}_{i}\right)^{D}\right)$ are finite (see [M2, Th. I.2.1]).

\subsection{Gluing dualities}

As was mentioned in the introduction of this section, we will need the following technical result to ensure that the completions of Proposition 4.1.iv. do not disturb the exact rows of the diagram (26) in any essential way. Then the proof of Theorem 4.3 is a matter of diagram chasing.

Lemma 4.2. Let $X$ be a smooth and proper geometrically irreducible variety over a $p$-adic field $K$. Consider the compatible system of pairings (26).

(i) The boundary map $H^{1}\left(K, \mathscr{G}_{i}\right) \rightarrow H^{2}\left(K, \mathscr{F}_{i-1}\right)$ has finite image for $i=1,2$ and is zero for $i \neq 1,2$.

(ii) The boundary map $H^{0}\left(K, \mathscr{F}_{i-1}^{D}\right) \rightarrow H^{1}\left(K, \mathscr{G}_{i}^{D}\right)$ has finite image for $i=1,2$ and is zero for $i \neq 1,2$.

(iii) The boundary map $H^{-1}\left(K, \mathscr{F}_{i-1}^{D}\right) \rightarrow H^{0}\left(K, \mathscr{G}_{i}^{D}\right)$ has finite image for $i=3$ and is zero for $i \neq 3$.

PROOF. We will only treat the finiteness statements since the vanishing always happens for trivial reasons (i.e., either the source or the target of the map is zero).

(i) In the case $i=2$ we have the finitely generated group $H^{1}\left(K, \mathscr{G}_{2}\right)=H^{0}(K, N S(\bar{X}))$ mapping to the torsion group $H^{2}\left(K, \mathscr{F}_{1}\right)$, so the image is finite. For $i=1$ the target is $H^{2}\left(K, \mathscr{F}_{0}\right)=\operatorname{Br} K$, and the image under consideration is contained in the kernel of $\operatorname{Br}(K) \rightarrow$ $\operatorname{Br}(X)$, which is well-known to be finite. 
(ii) In the case $i=1$ we have $H^{0}\left(K, \mathscr{F}_{0}^{D}\right)=\mathbf{Z}$ mapping to the torsion group $H^{1}\left(K, \mathscr{G}_{i}^{D}\right)=$ $H^{1}\left(K, \operatorname{Pic}^{0}(X / K)\right)$, so the image is finite. For $i=2$ we consider the commutative diagram

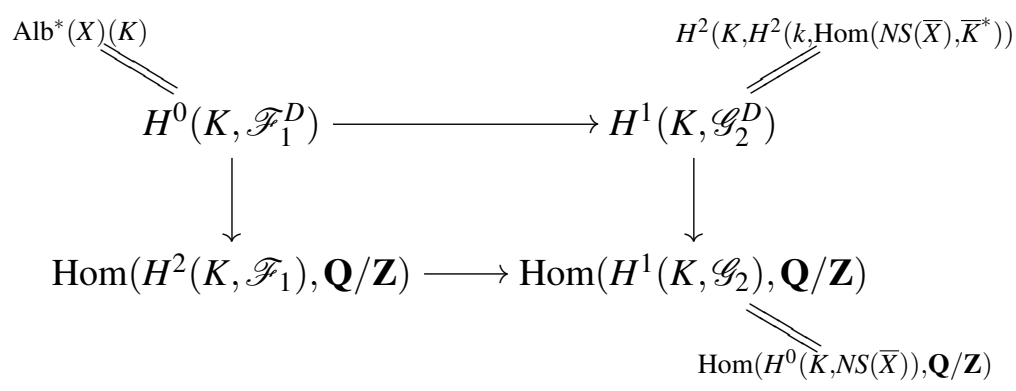

obtained from the system of pairings (26). The right hand vertical arrow is an isomorphism by Proposition 4.1, and the image of the bottom arrow is finite by case $i=2$ of part (i) of this lemma.

(iii) Observe that we consider the map

$$
H^{0}\left(K, \operatorname{Hom}\left(N S(\bar{X}), \bar{K}^{*}\right)\right) \rightarrow H_{\text {cont }}^{2}(K, \operatorname{Hom}(\operatorname{Br}(\bar{X}), \mathbf{Q} / \mathbf{Z})) .
$$

Since the target is a profinite group, it is sufficient to show that the image is torsion. Hence it is sufficient to show that the cokernel of the natural map

$$
{ }^{1} H_{1}(X, \mathbf{Z})=H^{-1}\left(K, \mathscr{F}_{\infty}\right) \rightarrow H^{-1}\left(K, \mathscr{F}_{2}^{D}\right)=H^{0}\left(K, \operatorname{Hom}\left(N S(\bar{X}), \bar{K}^{*}\right)\right) .
$$

is torsion. We will finish the proof by showing that this is a rather straightforward consequence of the fact that for divisors algebraic equivalence modulo torsion coincides with numerical equivalence.

By a trace argument it is sufficient to prove that the cokernel of the map

$$
\varepsilon:{ }^{1} H_{1}\left(X_{L}, \mathbf{Z}\right) \rightarrow H^{0}\left(L, \operatorname{Hom}\left(N S(\bar{X}), \bar{K}^{*}\right)\right)
$$

is torsion for some finite extension $L$ of $K$ in $\bar{K}$. We choose $L$ large enough such that $N S\left(X_{L}\right)=N S(\bar{X})$ and such that we have a finite collection

$$
f_{i}: C_{i} \rightarrow X_{L}
$$

of smooth, projective, geometrically irreducible curves $C_{i}$ over $L$ mapping to $X_{L}$, that generates a subgroup of finite index in $\operatorname{Hom}\left(N S\left(X_{L}\right), \mathbf{Z}\right)$ via the intersection product. To be precise, taking $\mathscr{Z}_{1}\left(\cup C_{i}\right)$ to be the group of 1 -dimensional cycles on the disjoint union of the $C_{i}$ we have that the right kernel of the pairing

$$
\begin{aligned}
& \mathscr{Z}_{1}\left(\bigcup C_{i}\right) \quad \times \quad N S\left(X_{L}\right) \rightarrow \quad \mathbf{Z} \\
& \sum a_{i}\left[C_{i}\right] \times[D] \quad \longmapsto a_{i}\left(f_{i}\right)_{*}\left[C_{i}\right] \cdot[D] \cdot
\end{aligned}
$$

is precisely the (finite) torsion subgroup of $N S\left(X_{L}\right)$.

After tensoring with $L^{*}$ we obtain a map

$$
\mathscr{Z}_{1}\left(\bigcup C_{i}\right) \otimes L^{*} \rightarrow \operatorname{Hom}\left(N S\left(X_{L}\right), L^{*}\right)
$$

of which the cokernel is torsion. By Section 2.2 we have a canonical isomorphism

$$
\mathscr{Z}_{1}\left(\bigcup C_{i}\right) \otimes L^{*}={ }^{1} H_{1}\left(\bigcup C_{i}, \mathbf{Z}\right),
$$


which fits into the following commutative diagram by the projection formula.

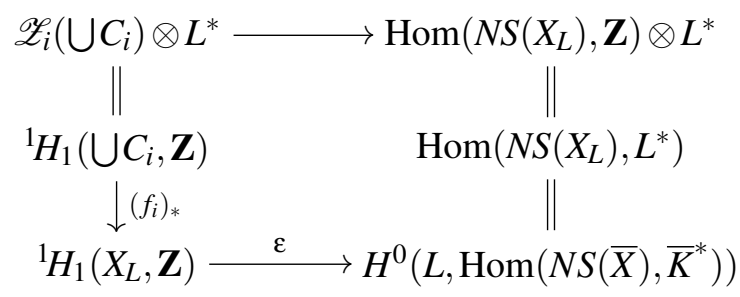

Hence the cokernel of the bottom arrow is torsion.

Theorem 4.3. Let $X$ be a nonsingular complete variety over a $p$-adic field $K$ For $i \geq 0$, let $\mathscr{F}_{i}$ be the complex defined in Section 2.2, and consider the Yoneda pairing

$$
H^{r}\left(K, \mathscr{F}_{i}^{D}\right) \times H^{2-r}\left(K, \mathscr{F}_{i}\right) \rightarrow H^{2}\left(K, \mathbf{G}_{m}\right)=\mathbf{Q} / \mathbf{Z} .
$$

(i) For every $i \geq 0$ and $r \in \mathbf{Z}$ the above pairing is nondegenerate.

(ii) For every $i \geq 0, r \leq-2$ the pairing is a perfect pairing between a profinite group and a torsion group.

(iii) For every $i \geq 0$ the induced pairings

$$
\begin{aligned}
& H^{2}\left(K, \mathscr{F}_{i}^{D}\right) \times H^{0}\left(K, \mathscr{F}_{i}\right)^{\curlywedge} \rightarrow \mathbf{Q} / \mathbf{Z}, \\
& H^{1}\left(K, \mathscr{F}_{i}^{D}\right) \times H^{1}\left(K, \mathscr{F}_{i}\right)^{\curlywedge} \rightarrow \mathbf{Q} / \mathbf{Z},
\end{aligned}
$$

are perfect pairings between a torsion group and a profinite group, and the pairings

$$
\begin{aligned}
H^{0}\left(K, \mathscr{F}_{i}^{D}\right)^{\wedge} \times H^{2}\left(K, \mathscr{F}_{i}\right) & \rightarrow \mathbf{Q} / \mathbf{Z}, \\
H^{-1}\left(K, \mathscr{F}_{i}^{D}\right)^{\wedge} \times H^{3}\left(K, \mathscr{F}_{i}\right) & \rightarrow \mathbf{Q} / \mathbf{Z} .
\end{aligned}
$$

are perfect pairings between a profinite group and a torsion group.

(iv) For every $i \geq 0, r>2$, the cohomology groups in the pairing are zero.

PROOF. In all four cases the proof will proceed by induction on the level $i$ of the 'filtration' $\mathscr{F}_{i}$, using Proposition 4.1 and Lemma 4.2 and the following commutative diagrams with exact rows that are obtained from the system of pairings (26).

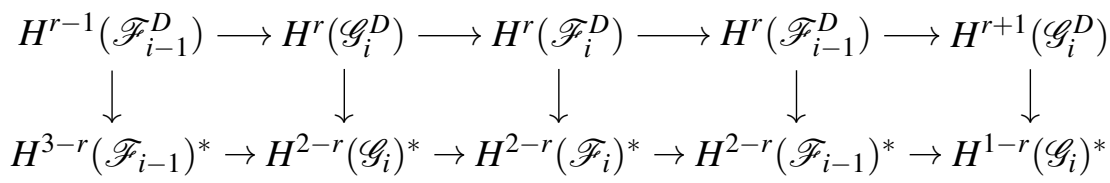

and

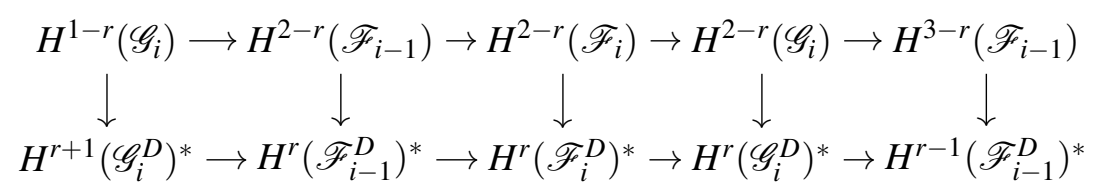

Here $H^{q}(-)$ is short for $H^{q}(K,-)$, and $-^{*}$ denotes the Pontryagin dual $\operatorname{Hom}_{\text {cont }}(-, \mathbf{Q} / \mathbf{Z})$. The exactness of the bottom rows is clear at the duals of torsion groups (which are equipped with the discrete topology); at the duals of groups which are not torsion (hence equipped with the profinite topology), the exactness follows from [M2, Prop. 0.20] and Lemma 4.2. 
(i) In order to show that $H^{r}\left(\mathscr{F}_{i}^{D}\right) \rightarrow H^{2-r}\left(\mathscr{F}_{i}\right)^{*}$ is injective, consider diagram (27). By the induction hypothesis and Proposition 4.1, we know that all vertical arrows but the middle one are injective. In the leftmost column, we have for $r \neq 0,1$ that the vertical map $H^{r-1}\left(\mathscr{F}_{i-1}^{D}\right) \rightarrow H^{3-r}\left(\mathscr{F}_{i-1}\right)^{*}$ is an isomorphism. For $r \in\{0,1\}$ we have that the image of the horizontal map $H^{r-1}\left(\mathscr{F}_{i-1}^{D}\right) \rightarrow H^{r}\left(\mathscr{G}_{i}^{D}\right)$ is a finite (possibly trivial) group $I$ by Lemma 4.2, so then we may replace the left column of diagram (27) by the isomorphism $I \rightarrow I^{*}$. The injectivity now follows by a diagram chase. The map $H^{2-r}\left(\mathscr{F}_{i}\right) \rightarrow H^{r}\left(\mathscr{F}_{i}^{D}\right)^{*}$ is treated similarly.

(ii) For $r \leq-2$ the surjectivity of the maps $H^{r}\left(\mathscr{F}_{i}^{D}\right) \rightarrow H^{2-r}\left(\mathscr{F}_{i}\right)^{*}$ and $H^{2-r}\left(\mathscr{F}_{i}\right) \rightarrow$ $H^{r}\left(\mathscr{F}_{i}^{D}\right)^{*}$ follows by a similar diagram chase, using Proposition 4.1 and the induction hypothesis, which give the surjectivity of the second and the fourth vertical arrows and the injectivity of the rightmost vertical arrow in diagrams (27) and (28).

(iii) The injectivity and surjectivity of the map

$$
H^{0}\left(\mathscr{F}_{i}\right)^{\wedge} \rightarrow H^{2}\left(\mathscr{F}_{i}^{D}\right)^{*}
$$

follows immediately from Proposition 4.1 , since $H^{0}\left(\mathscr{F}_{i}\right)=H^{0}\left(\mathscr{G}_{0}\right)$ and $H^{2}\left(\mathscr{F}_{i}^{D}\right)=$ $H^{2}\left(\mathscr{G}_{0}^{D}\right)$ for all $i \geq 0$. The injectivity and surjectivity of

$$
H^{1}\left(\mathscr{F}_{i}\right)^{\wedge} \rightarrow H^{2}\left(\mathscr{F}_{i}^{D}\right)^{*}
$$

follows by induction from diagram (28) with $r=1$, Proposition 4.1, and Lemma 4.2, since we may replace the right hand column $H^{2}\left(\mathscr{F}_{i-1}\right) \rightarrow H^{0}\left(\mathscr{F}_{i-1}^{D}\right)^{*}$ of the diagram by finite groups, and then the upper row remains exact after taking profinite completions. For the isomorphisms $H^{0}\left(\mathscr{F}_{i}^{D}\right)^{\curlywedge} \rightarrow H^{2}\left(\mathscr{F}_{i}\right)^{*}$ and $H^{-1}\left(\mathscr{F}_{i}^{D}\right)^{\curlywedge} \rightarrow H^{3}\left(\mathscr{F}_{i}\right)^{*}$ we use similar arguments.

For the arrows in the other direction, like

$$
H^{2}\left(\mathscr{F}_{i}^{D}\right) \rightarrow\left(H^{0}\left(\mathscr{F}_{i}\right)^{\uparrow}\right)^{*}=H^{0}\left(\mathscr{F}_{i}\right)^{*}
$$

we have injectivity by part (i) of this theorem, and the surjectivity follows by diagram chasing in (27) and (28) and induction on $i$ from Proposition 4.1.

(iv) This follows from Proposition 4.1.iv by induction on $i$.

\subsection{The period of $X$ and the Picard group}

Using Theorem 4.3 we will now prove the result mentioned in introduction relating the period $P(X)$ of a smooth, proper variety $X$ over a $p$-adic field $K$ to the cokernel of the map $\operatorname{Pic}^{0}(X) \rightarrow \operatorname{Pic}^{0}(X / K)(K)$.

Proposition 4.4. Let $X$ be a smooth proper irreducible variety over a $p$-adic field $K$. The cokernel of the natural map

$$
\operatorname{Pic}^{0}(X) \rightarrow \operatorname{Pic}^{0}(X / K)(K)
$$

is a finite cyclic group of order equal to the period $P(X)$ of $X$.

PROOF. With notations as before, consider the following triangle in the derived category of sheaves on $k_{\mathrm{sm}}$ :

$$
\mathscr{F}_{0} \rightarrow \mathscr{F}_{1} \rightarrow \mathscr{G}_{1} \rightarrow \mathscr{F}_{0}[1]
$$

Recall from Section 2.2 that $\mathscr{F}_{0}=\mathbf{G}_{m}$, and $\mathscr{G}_{1}=\operatorname{Pic}^{0}(X / K)[-1]$. It can be checked that $\operatorname{Pic}^{0}(X)=H^{1}\left(K, \mathscr{F}^{1}\right)$. Taking Cartier duals, we get the triangle

$$
\operatorname{Alb}(X) \rightarrow \operatorname{Alb}^{*}(X) \rightarrow \mathbf{Z} \rightarrow \operatorname{Alb}(X)[1]
$$


(See Section 2.2 and Theorem 3.6).

We will show that the cokernel of

$$
\operatorname{Pic}^{0}(X)=H^{1}\left(K, \mathscr{F}_{1}\right) \rightarrow H^{1}\left(K, \mathscr{G}_{1}\right)=\operatorname{Pic}^{0}(X / K)(K)
$$

is dual to the cokernel of

$$
\operatorname{Alb}^{*}(X)(K) \rightarrow \mathbf{Z}
$$

The cokernel of $H^{1}\left(K, \mathscr{F}_{1}\right) \rightarrow H^{1}\left(K, \mathscr{G}_{1}\right)$ coincides with the kernel of $\operatorname{Br}(K)=$ $H^{2}\left(K, \mathscr{F}_{0}\right) \rightarrow H^{2}\left(K, \mathscr{F}_{1}\right)$, so we consider the following diagram of compatible pairings that we obtain from the Yoneda pairing:

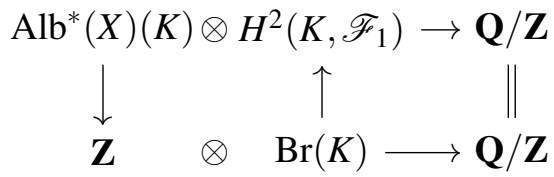

The pairings are nondegenerate by Theorem 4.3.

\section{References}

[SGA4] M. Artin, A. Grothendieck, and J.-L. Verdier (eds.), Théorie des topos et cohomologie étale des schémas (SGA 4), tome 3, Lect. Notes Math. 305, Springer-Verlag, 1973.

[B1] S. Bloch, Lectures on algebraic cycles, Duke University Mathematics Department, 1980.

[Br1] L. S. Breen, On a nontrivial higher extension of representable abelian sheaves, Bull. Amer. Math. Soc. 75 (1969), 1249-1253.

[Br2] L. S. Breen, Extensions of abelian sheaves and Eilenberg-MacLane algebras, Invent. Math. 9 (1969), $15-44$.

[CTS] J.-L. Colliot-Thélène and S. Saito, Zéro-cycles sur les variétés p-adiques et groupe de Brauer, Int. Math. Res. Not. (1996), 151-160.

[SGA4.5] P. Deligne, Cohomologie étale (SGA 4 1/2), Lect. Notes Math. 569, Springer-Verlag, 1977.

[Gr1] A. Grothendieck, Fondements de la géométrie algébrique. Extraits du Séminaire Bourbaki, 19571962, Secrétariat mathématique, Paris, 1962.

[Gr2] A. Grothendieck, Le groupe de Brauer I, II, III, in: Dix exposés sur la cohomologie des schémas, Advanced Studies in Pure Math. 3, North-Holland, Masson, 1968, pp. 46-188.

[J] U. Jannsen, Continuous étale cohomology, Math. Ann. 280 (1988), 207-245.

[K] B. Kahn, A sheaf-theoretic reformulation of the Tate conjecture, electronic preprint http://arxiv.org/ abs/math.AG/9801017, 1998.

[La] G. Laumon, Homologie étale, Exp. VIII in: Séminaire de Géometrie Analytique (A. Douady and J.-L. Verdier, eds.), Astérisque 36-37, 1974-1975, pp. 163-188.

[L1] S. Lichtenbaum, Duality theorems for curves over p-adic fields, Invent. Math. 7 (1969), 120-136.

[L2] S. Lichtenbaum, Values of zeta-functions at nonnegative integers, in: (H. Jager, ed.) Number theory (Noordwijkerhout, 1983), Lect. Notes Math. 1068, Springer-Verlag, 1984, pp. 127-138.

[M1] J. S. Milne, Étale cohomology, Princeton University Press, 1980.

[M2] J. S. Milne, Arithmetic duality theorems, Perspectives in mathematics 1, Academic Press, 1986.

[Mum] D. Mumford, Abelian Varieties, Tata Institute of Fundamental Research Studies in Mathematics 5, Oxford University Press, 1970.

[Mur] J. P. Murre, On contravariant functors from the category of pre-schemes over a field into the category of abelian groups (with an application to the Picard functor), Inst. Hautes Études Sci. Publ. Math. 23 (1964), 5-43.

[O] F. Oort, Commutative group schemes, Lect. Notes Math. 15, Springer-Verlag, 1966.

[PS] R. Parimala and V. Suresh, Zero-cycles on quadric fibrations: Finiteness theorems and the cycle map, Invent. Math. 122 (1995), 83-117.

[Ra] N. Ramachandran, Duality of Albanese and Picard 1-motives, K-Theory 22 (2001), 271-301.

[Ro] A.A. Rojtman, The torsion of the group of 0 -cycles modulo rational equivalence, Ann. of Math. (2), 111 (1980), 553-569. 
[Ro] P. Roquette, Splitting of algebras by function fields of one variable, Nagoya Math. J. 27 (1966), 625-642.

[Sa] S. Saito, A global duality theorem for varieties over global fields, in: (J. F. Jardine, V. P. Snaith, eds.) Algebraic $K$-theory: connections with geometry and topology (Lake Louise, 1987), 425-444, Kluwer Acad. Publ., 1989

[Se] J.-P. Serre, Cohomologie galoisienne, 5th ed., Lect. Notes Math. 5, Springer-Verlag, 1994.

[vH1] J. van Hamel, Divisors on real algebraic varieties without real points, Manuscripta Math. 98 (1999), 409-424.

[vH2] J. van Hamel, The Brauer-Manin obstruction for zero-cycles on Severi-Brauer fibrations over curves, J. London Math. Soc. (2) 68 (2003), 317 - 337.

[vH3] J. van Hamel, The Reciprocity obstruction for rational points on compactifications of torsors under tori over fields with global duality, Documenta Math. 8 (2003), 125-142.

School of Mathematics and Statistics F07, University of Sydney, NSW 2006, Australia

E-mail address: vanhamel@member.ams .org 